\title{
Oscillating blue stragglers, $\gamma$ Doradus stars and eclipsing binaries in the open cluster NGC 2506 ${ }^{\star} \star \star$
}

\author{
T. Arentoft ${ }^{1,2}$, J. De Ridder ${ }^{3}$, F. Grundahl ${ }^{1,2}$, L. Glowienka ${ }^{1}$, C. Waelkens ${ }^{3}$, M.-A. Dupret ${ }^{4}$, A. Grigahcène ${ }^{5}$, \\ K. Lefever ${ }^{3}$, H. R. Jensen ${ }^{1}$, M. Reyniers ${ }^{3}$, S. Frandsen ${ }^{1,2}$, and H. Kjeldsen ${ }^{1,2}$ \\ 1 Department of Physics and Astronomy, University of Aarhus, 8000 Aarhus C., Denmark \\ e-mail: toar@phys.au.dk \\ 2 Danish AsteroSeismology Centre, University of Aarhus, 8000 Aarhus C., Denmark \\ Instituut voor Sterrenkunde, Katholieke Universiteit Leuven, 3001 Heverlee, Belgium \\ ${ }^{4}$ LESIA, Observatoire de Paris-Meudon, UMR 8109, 92190 Meudon, France \\ 5 GRAAG, Algiers Observatory, BP 63 Bouzareah, 16340 Algiers, Algeria
}

Received 14 December 2006 / Accepted 25 January 2007

\section{ABSTRACT}

\begin{abstract}
Context. This is the first step in a project to combine studies of eclipsing binaries and oscillating stars to probe the interior of Blue Stragglers (BS). This may imply a way to discriminate observationally between different birth mechanisms of BS stars.

Aims. We study the open cluster NGC 2506 which contains oscillating BS stars and detached eclipsing binaries for which accurate parameters can be derived. This will tightly constrain the cluster isochrone and provide an absolute mass, radius and luminosity-scale for the cluster stars along with the cluster age, metallicity and distance. The present work focuses on obtaining the light curves of the binaries and determine their orbital periods, on obtaining power spectra of the oscillating BS stars to select targets for follow-up studies, and on searching for $\gamma$ Doradus type variables which are also expected to be present in the cluster.

Methods. With a two-colour, dual-site photometric campaign we obtained 3120 CCD-images of NGC 2506 spread over four months. We analysed the $B I$ time-series of the oscillating stars and used simulations to derive statistical uncertainties of the resulting frequencies, amplitudes and phases. A preliminary mode-identification was performed using frequency ratios for the oscillating BS stars, and amplitude ratios and phase differences for a population of newly detected $\gamma$ Doradus stars.

Results. We quadrupled the number of known variables in NGC 2506 by discovering 3 new oscillating BS stars, $15 \gamma$ Doradus stars and four new eclipsing binaries. The orbital periods of 2 known, detached eclipsing binaries were derived. We discovered a BS star with both p-mode and g-mode variability and we confronted our $\gamma$ Doradus observations with state-of-the-art seismic models, but found significant discrepancy between theory and observations.

Conclusions. NGC 2506 is an excellent target for asteroseismic tests of stellar models, as strong external constraints can be imposed on the models of a population of more than 20 oscillating stars of different types.
\end{abstract}

Key words. stars: blue stragglers - stars: variables: delta Sct, $\gamma$ Dor - stars: binaries: eclipsing galaxies: open clusters and associations: individual: NGC 2506 - techniques: photometric

\section{Introduction}

Blue straggler (BS) stars are still a much debated phenomenon. In a cluster HR diagram, these stars are situated above and blueward of the main sequence (MS) turn-off point, where more massive stars were located before they evolved away to red giants. Their appearance in a region of the HR diagram where they should not be contradicts our standard single-star evolution theory.

Although the origin of BS stars is still not fully understood, the generally accepted working hypothesis is that these stars originate from stellar collisions, binary coalescence, or from mass transfer in a binary system. These ideas go back to the pioneering work of Hills \& Day (1976), and McCrea (1964).

* Based on observations obtained at the Flemish Mercator telescope on La Palma, Spain, the Danish 1.5-m telescope at ESO, La Silla, Chile, and on observations collected at the European Southern Observatory, Paranal, Chile (ESO Programme 075.D-0206(B)).

$\star \star$ Catalog of individual photometry measurements for all variables is only available in electronic form at the CDS via anonymous ftp to cdsarc.u-strasbg.fr $(130.79 .128 .5)$ or via

http://cdsweb.u-strasbg.fr/cgi-bin/qcat?J/A+A/465/965
Because of these stellar interactions, a BS star is formed with a mass greater than the turn-off mass of the cluster. Such a star is therefore able to prolong its life on the MS, or even reappear as a born-again star, which explains why BS stars have not yet evolved far away from the MS.

However, before this hypothesis can be promoted to a theory, more observational and theoretical evidence needs to support and quantify it, and this is what current BS research is focusing on. We mention the recent result of Ferraro et al. (2004) who found a bimodal radial BS population distribution in the globular cluster 47 Tuc, which they interpreted as evidence for different but simultaneously working BS birth mechanisms (see also Mapelli et al. 2006, for a follow-up article). For the same cluster Ferraro et al. (2006) pointed out that at least some of the BS stars seem to show the chemical signature of binary mass transfer, adding another piece of evidence for the mass transfer scenario. Progress on understanding the collisional scenario has mainly been made on the theoretical side. Sills et al. (2001, 2005) used smoothed particle hydrodynamics simulations to show that off-axis collision products have rotational velocities well above their break-up velocity so that they can only survive if they can 
somehow get rid of the excess angular momentum. The authors mention magnetic breaking caused by a circumstellar disk as a possible cause of a BS spin down. Such discs may (De Marco et al. 2004) or may not (Porter \& Townsend 2005) already have been discovered around BS stars.

Clearly, the theories for the different BS birth mechanisms are getting more established through the increasing amount of evidence. It's still difficult, though, to recognize how a particular BS star was born. Especially a diagnostic to discriminate observationally between binary coalescence and stellar collision is not available at the moment. This makes it difficult to study, for example, to what extent and for how long the interior of a BS star formed by "unrolling" a donor star is different from one formed by a direct collision of two stars.

Might asteroseismology help in this respect? In many clusters the BS stars are situated in the classical instability strip where one expects $\delta$ Scuti or SX Phe oscillations. Many oscillating BS stars have indeed already been detected, see for example Gilliland \& Brown (1992), Gilliland et al. (1998). These oscillations could provide a way to model the BS star interior. The fact that the BS stars are part of a cluster makes them even more suitable asteroseismic targets, as chemical composition and age may be assumed constant for each BS member. These constraints can be made quantitatively more stringent if the cluster also contains detached eclipsing binaries for which accurate masses and radii can be determined, so that the isochrone can be precisely calibrated (see Grundahl et al. 2006). Finally, a combined set of observed frequencies in a number of oscillating stars within the same cluster, can be used to extract more information than for single stars (Frandsen \& Kjeldsen 1993).

This paper reports on the first stage of this Blue Straggler project: the challenging task of deriving useful oscillation spectra of BS stars in the open cluster NGC 2506. The reason for choosing NGC 2506 is fourfold. First, the cluster contains BS stars of which three of them were shown to oscillate by Kim et al. (2001), by means of photometric time series (V1V3). Secondly, the cluster contains detached eclipsing binaries. One was found by Kim et al. (2001, V4), and another by ourselves (V5). Thirdly, it is an open cluster close to the celestial equator so that it is visible from both hemispheres, making a multi-site campaign of variable stars possible. Finally, most of the cluster is confined within a radius of 6 arcmin, making it fit nicely in a typical CCD image; yet the cluster is not so crowded that the photometric precision is affected.

Besides the BS project, we present another project which started as a side result but which has meanwhile grown into an equally important project. The turn-off stars of NGC 2506 span a large part of the $\gamma$ Doradus instability strip making the cluster perfect for investigating the $\gamma$ Doradus phenomenon, should such stars be present. Kim et al. (2001) searched for $\gamma$ Doradus stars without detecting any. The data presented here are both more extensive and have a higher precision per data point, making the search more sensitive and, as it turns out, more successful because we do detect a surprisingly high number of $\gamma$ Doradus stars in the cluster. These $\gamma$ Doradus stars are very promising asteroseismic objects as their high-order g-modes probe deeply in the stellar interior. In addition, they are exciting laboratories to study stellar physics such as semi-convection, convective overshooting, and (both slow and fast) stellar rotation.

Only recently, however, could some quantitative comparison between theory and observations be carried out for the $\gamma$ Doradus stars. Dupret et al. (2004, 2005a) explained the driving mechanism of the $\gamma$ Doradus g-modes by using the time-dependent convection (TDC) treatment of Grigahcéne et al. (2005). With the same TDC models, Dupret et al. (2005b) explained for the first time and with success the photometric amplitude ratios and phase differences of five field $\gamma$ Doradus stars. In the same way as for the BS stars, our cluster $\gamma$ Doradus stars provide a more stringent test of the theory. This paper reports the discovery of many new $\gamma$ Doradus stars, the observed frequencies, and a first attempt to model the photometric amplitude ratios and phase differences with the TDC theory, with remarkable results.

\section{The target cluster: NGC 2506}

NGC $2506\left(\alpha_{2000}, \delta_{2000}=08^{\mathrm{h}} 00^{\mathrm{m}} 01^{\mathrm{s}},-10^{\circ} 46^{\prime} 12^{\prime \prime}\right)$ is an old open cluster that already received quite some attention in the literature. The morphology study of Chen et al. (2004) indicates that it is a mildly elongated cluster counting about 1091 stars, and the recently published catalogue of Kharchenko et al. (2005) puts NGC 2506 at a distance of 3460 pc. There seems to be a consensus in the literature about the age of the cluster, which would be $t=2.14 \pm 0.35$ Gyr (Salaris et al. 2004) although Xin \& Deng (2005) quotes an age of 3.4 Gyr. Such an old age is inconsistent with our Strömgren photometry which also points to an age close to 2 Gyr. We refer to Carretta et al. (2004) for a literature overview on the metallicity of NGC 2506. Although the different values vary quite a lot, all chemical analyses show that the cluster is metal deficient. We give a somewhat higher weight to the value obtained by Carretta et al., $[\mathrm{Fe} / \mathrm{H}]=-0.20 \pm 0.01$, as it is the only metallicity obtained through an iron abundance analysis using high-resolution spectroscopy. Nevertheless, we treat their quoted error bar as a lower limit, as their average metallicity value is based on a sample of only 2 stars. Also, their value differs from other values reported in the literature obtained from photometry or low-resolution spectroscopy. Gratton (2000), for example, lists a value of $[\mathrm{Fe} / \mathrm{H}]=-0.42 \pm 0.09$. This leads us to believe that the uncertainty on the metallicity is actually larger than 0.1 dex. As a final cluster parameter, we adopt the reddening given by Carretta et al. (2004): $E(B-V)=0.073 \pm 0.009$.

\section{The data and the data reduction}

Our photometric time-series CCD observations were obtained using DFOSC at the Danish 1.54-m telescope at ESO, La Silla, and MEROPE at the Flemish 1.2-m Mercator telescope at La Palma. At ESO we observed during 23 nights in January 2005, at La Palma during 20 nights in the period January-April 2005. Overlapping data were obtained on some of the January nights.

In order to optimise the time series to the highest possible precision, care was taken to maintain the same pointing during the observations, i.e., to keep the stars at fixed pixel positions on the CCD. This was done by starting each night with the same pointing as the previous nights (within a few pixels) and keeping this pointing using autoguiding. The exposure times were chosen so that the brightest of the known variables, V1, were below the saturation limit. At ESO, this led to exposure times of approximately $100 \mathrm{~s}$ in $B$ and $30 \mathrm{~s}$ in $I$, hence sequences of $(2 B, 4 I, 2 B, \ldots)$ were obtained. At the Mercator, longer exposure times were possible due to the smaller telescope aperture, but $120 \mathrm{~s}$ were used for both filters in order to maintain sufficient time resolution for the short-period oscillating BS stars.

In total, $2100 B I$ images were obtained at ESO, and 1020 at La Palma, corresponding to $101.8(B)$ and $97.8(I)$ hours of observing time at ESO, and $60.9(B)$ and $60.4(I)$ at La Palma. In Fig. 1 all data are shown for the variable V3, illustrating the time distribution of the data set. Most of the data were obtained 


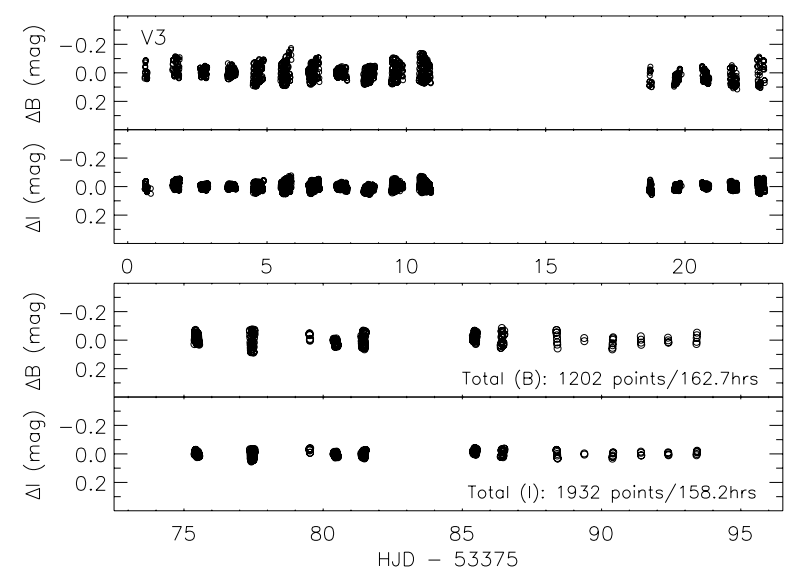

Fig. 1. The total data set for the variable V3, showing the time distribution of the combined data from the two sites (ESO and La Palma).

in January 2005 (136/132 h for $B / I)$ with additional data obtained in March and April at the Mercator. In the gap between the first and second batch of observations (Fig. 1, upper panels) a few data points were obtained per night at La Silla, to monitor the EBs. These data are not included in the analysis of the oscillating stars presented here. The data from March and April were obtained at higher airmass and had lower exposure levels (\#counts/star), resulting in higher noise per data point.

The CCD images were calibrated using standard procedures. The mean BIAS level was subtracted from each frame using overscan areas, and a median BIAS image (with zero mean) was then subtracted. Evening and morning sky-flats had been obtained whenever possible, and from those, a single, median sky-flat were constructed per filter and per observing run. The run at the D1.5-m spans nearly a month, but before applying the flat-field calibration to the science images, we checked that the flat-fields were indeed stable over the length of the observing run.

The photometric reductions were done separately for the Mercator and ESO data, using the software package MOMF (Kjeldsen \& Frandsen 1992). MOMF applies a very robust algorithm combining PSF and aperture photometry, and is excellent for time-series photometry in semi-crowded fields. The data from each site were merged on a star-by-star basis, by shifting the mean levels of the individual light curves to zero. An example of this merging is shown in Fig. 2 for V3 during a single night. Not all stars were present in both data sets; due to differences in field-of-view (MEROPEs FOV is 6.5 × 6.5, DFOSCs $\left.15^{\prime} \times 15^{\prime}\right)$ more stars were observed at ESO. The observed field is shown in Fig. 20 (a DFOSC image), which also serves as a finding chart for the variable stars discussed below.

\subsection{Analysis of differential light curves}

The output from MOMF is differential time series photometry for all stars in the images, using the mean of all stars as the reference level to take out atmospheric effects. The light curves were kept in the instrumental system, but calibrated Strömgren photometry has previously been obtained for the cluster by F. Grundahl; these data are presently unpublished, but calibrated as described in Grundahl et al. (2002). We will in the present paper rely on these data for deriving stellar parameters for the variable stars.

We obtained light curves for 863 stars in the MEROPE field and 3354 stars in the DFOSC field, of which one must expect

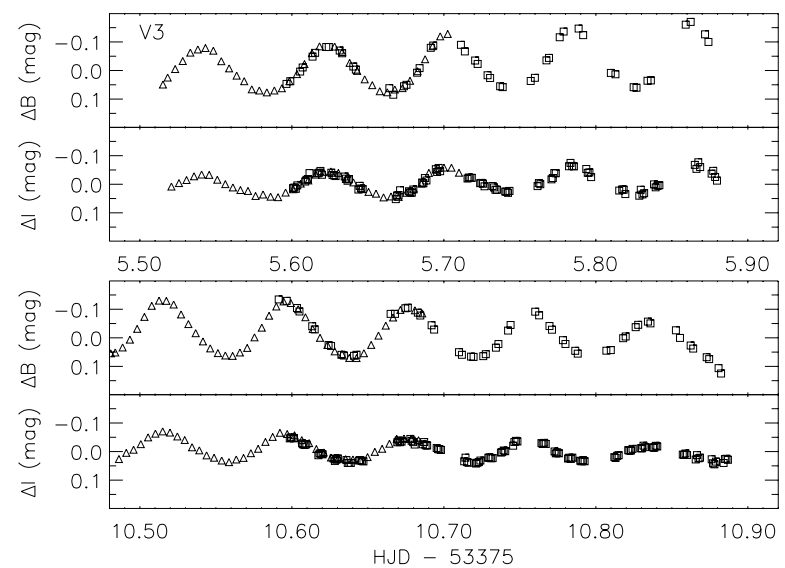

Fig. 2. Overlapping data on V3. Triangles are data from La Palma, squares are from La Silla. A slow trend with a frequency of $1.0 \mathrm{~d}^{-1}$ was present in the La Silla $B$-data only, and was subtracted before merging the data (see Sects. 3.1 and 4.1).

that a significant fraction, especially of those far from the cluster center, are not physical members of the cluster. The resulting photometric precision in the light curves was as aimed for, with a precision per data point of 1-2 mmag for the bright stars (on good nights), gradually decreasing for stars of fainter magnitudes as the photon noise increases.

The aim of our data analysis was to detect all variable stars in the observed fields, and to use all data for a detailed analysis of the variability. The first step in the analysis was to clean all light curves for obvious outliers and to cross-identify the common stars in the MEROPE and DFOSC fields. We then calculated amplitude spectra for all stars, based on the MEROPE and DFOSC data separately and inspected the light curves and amplitude spectra of each star in the two data sets manually, to select possible variable stars for further analysis. For stars to be selected as candidates, we required that variability was present in both the MEROPE and DFOSC data (for common stars) and in both the $B$ - and $I$ filter data.

At this stage we had a list of 33 variable star candidates, of which 28 ended up being retained as variables, while 5 stars were rejected at a later stage in the analysis. For the candidates observed at both telescopes we now merged the MEROPE and DFOSC data by shifting the light curves to zero mean. For some of the blue stragglers (such as V3; see Fig. 2), we subtracted some incoherent, low-frequency variations in order to match up the data from the two sites. As these stars are bluer than the large majority of cluster stars, we ascribe these variations to secondorder extinction effects that remain uncorrected because the differential photometry is dominated by the redder cluster turn-off stars.

Because the data quality during a time series is variable, we assigned statistical weights to each data point (see e.g., Arentoft et al. 1998; Handler 2003). This was done by highpass filtering the time series, using the Fourier transform, so that the filtered light curve only contained high-frequency (white) noise, which is useful for finding deviating points. We tried different weighting schemes based on the deviation of the individual data points from the light curve mean compared to the overall standard deviation $(\sigma)$ of the light curve. In the end we chose to give points deviating less than $2 \sigma$ in the highpass filtered series weight 1.0 , while points deviating more were weighted as $1 / \sigma^{2}$, as this scheme resulted in the lowest noise in the weighted amplitude spectra. 


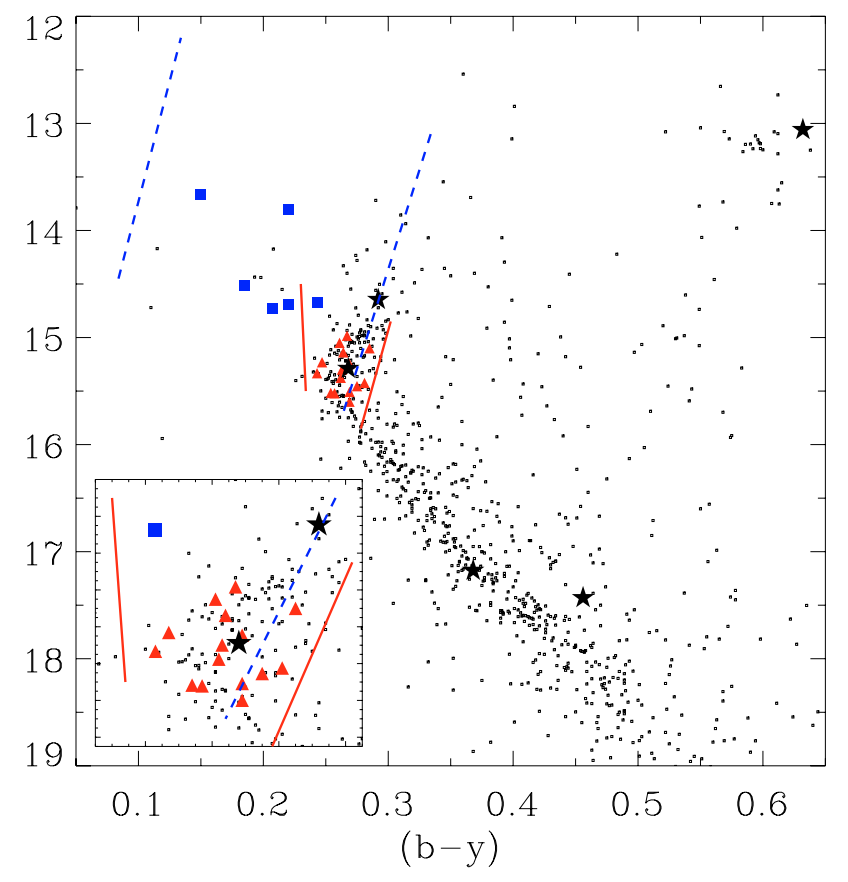

Fig. 3. Colour-magnitude diagram for NGC 2506 with the detected variables marked. The blue dashed lines are the observational $\delta$ Scuti instability strip (Breger 2000) and the red solid lines the $\gamma$ Doradus instability strip from Handler \& Shobbrook (2002). A distance modulus of 12.65 and $E(b-y)=0.054$ was used for placing the instability strips in the CMD. Blue squares marks the oscillating BS stars, red triangles the $\gamma$ Doradus candidates, and black stars the eclipsing binaries. The insert in the lower left corner shows a zoomed view of the $\gamma$ Dor instability strip. (Colours are only visible in the electronic version.)

We then scrutinized the evidence for variability, using weighted amplitude spectra as well as the light curves, for each of our 33 candidates. In this way we detected 28 variables, of which only 5 were previously known. The variables, which will be discussed in detail below, are 6 oscillating blue straggler stars ( $\delta$ Scuti oscillations), $15 \gamma$ Doradus candidates, 6 binaries and one star of presently unknown type. The classification is based on the time scale of the variability ( $\sim$ days for the $\gamma$ Doradus candidates, $\sim$ hours for the oscillating blue stragglers), BI amplitude ratios - to separate $\gamma$ Doradus stars from, e.g., ellipsoidal variables - as well as the position of the stars in the colour-magnitude diagram. Figure 3 plots this diagram based on the Strömgren data mentioned above, with the detected variables and the observational $\delta$ Scuti (Breger 2000) and $\gamma$ Doradus (Handler \& Shobbrook 2002) instability strips indicated. In fact, we will in the following argue, that the majority of the $\gamma$ Doradus candidates, which are all positioned inside the instability strip, can be considered bona fide $\gamma$ Doradus stars (Handler 1999; Handler \& Shobbrook 2002).

The last step in the data analysis was a detailed frequency analysis of the oscillating stars, and a period search for the eclipsing binaries. For the oscillating stars, the frequency analysis was carried out using PERIOD98 (Sperl 1998), which uses simultaneous least-squares fits of all detected frequencies in a light curve. We only include frequencies that are present in both the $B$ and the $I$ data, with a Signal-to-Noise ratio in the amplitude spectra of 4 in at least one of the filters, although most detected frequencies meet this requirement for both filters. Because our $B$ and $I$ data have different sampling, the spectral window functions are slightly different for the two cases. This may lead to small differences in the detected frequency values between the
Table 1. Variability data for the oscillating BS stars. Frequencies are in $\mathrm{d}^{-1}$ and amplitudes are given in mmag. The quoted errors on the frequencies are standard deviations on the values determined from extensive simulations (see text).

\begin{tabular}{cccrrr}
\hline \hline ID & $v_{i}$ & $v$ & $A_{I}$ & $A_{B}$ & $S / N_{B}$ \\
\hline V1 & $v_{1}$ & $13.630(1)$ & 3.2 & 6.8 & 15.1 \\
& $v_{2}$ & $14.684(1)$ & 2.8 & 5.1 & 11.2 \\
& $v_{3}$ & $14.716(1)$ & 1.7 & 2.9 & 6.4 \\
& $v_{4}$ & $16.688(5)$ & 1.5 & 2.8 & 6.2 \\
& $v_{5}$ & $16.896(2)$ & 1.0 & 2.2 & 5.0 \\
& $v_{6}$ & $17.043(2)$ & 0.9 & 1.8 & 4.0 \\
& $v_{7}$ & $17.672(1)$ & 2.2 & 4.5 & 10.0 \\
V2 & $v_{1}$ & $10.854(1)$ & 55.8 & 121.4 & 215.0 \\
& $v_{2}$ & $20.141(1)$ & 7.5 & 14.2 & 29.5 \\
& $2 v_{1}$ & $21.709(1)$ & 8.5 & 17.0 & 35.3 \\
& $v_{3}$ & $30.995(2)$ & 1.2 & 2.8 & 5.9 \\
& $3 v_{1}$ & $32.563(3)$ & 1.3 & 3.1 & 6.4 \\
V3 & $v_{1}$ & $0.526(1)$ & 3.2 & 6.9 & 3.1 \\
& $v_{2}$ & $0.827(6)$ & 4.4 & 10.4 & 4.7 \\
& $v_{3}$ & $0.908(3)$ & 4.9 & 12.2 & 5.5 \\
& $v_{4}$ & $11.243(2)$ & 1.7 & 5.8 & 6.4 \\
& $v_{5}$ & $12.031(1)$ & 7.4 & 16.8 & 18.6 \\
& $v_{6}$ & $12.265(1)$ & 25.9 & 56.2 & 62.0 \\
& $v_{7}$ & $12.748(5)$ & 2.2 & 3.9 & 4.4 \\
& $v_{8}$ & $13.088(1)$ & 10.0 & 18.2 & 20.1 \\
& $v_{9}$ & $18.555(4)$ & 2.5 & 3.7 & 4.1 \\
& $v_{10}$ & $24.336(5)$ & 1.7 & 3.2 & 4.5 \\
& $v_{11}$ & $24.530(2)$ & 2.1 & 4.3 & 6.0 \\
V6 & $v_{1}$ & $9.441(3)$ & 0.9 & 3.7 & 5.9 \\
& $v_{2}$ & $10.767(1)$ & 5.1 & 10.9 & 17.2 \\
V7 & $v_{3}$ & $13.958(3)$ & 1.4 & 3.1 & 4.9 \\
V8 & $v_{1}$ & $10.576(1)$ & 6.9 & 15.3 & 22.3 \\
& $v_{1}$ & $10.227(2)$ & 2.9 & 4.5 & 4.5 \\
& $v_{2}$ & $10.611(1)$ & 4.4 & 11.1 & 11.2 \\
& $v_{3}$ & $10.781(1)$ & 5.0 & 11.7 & 11.7 \\
& $v_{4}$ & $11.550(2)$ & 4.7 & 8.3 & 8.4 \\
& $v_{5}$ & $16.149(2)$ & 3.4 & 6.8 & 6.8 \\
& $v_{6}$ & $19.423(2)$ & 3.4 & 7.2 & 7.2 \\
& $v_{7}$ & $20.944(3)$ & 1.8 & 3.4 & 3.4 \\
\hline & & & & & \\
& & & & \\
6
\end{tabular}

two filters. For each detected frequency, we therefore manually inspected the amplitude spectra, to find a frequency value that described the variation in both filters well. For each star, we then kept the detected frequencies fixed, and fitted all frequencies simultanously to the $B$ and $I$-band data separately, to determine amplitudes and phases for both filters.

All detected frequencies for the oscillating blue stragglers and the $\gamma$ Doradus stars are given in Tables 1 and 2, and Strömgren indices for all variables, as well as stellar parameters for most of these stars, have been collected in Table 3. We have used the calibration of Alonso et al. (1996) for deriving effective temperatures, the bolometric corrections follow Lejeune et al. (1998), and luminosities have been derived using the known cluster distance and $M_{\mathrm{bol}, \odot}=4.746$.

\subsection{Mode parameter uncertainties}

As we plan to use the amplitude ratios and phase differences for theoretical modeling of the $\gamma$ Doradus stars, we should have an idea how large the uncertainty is on the observed values. One possibility would be to use the well-known expressions for the standard errors of the amplitudes and phases given by e.g. Breger et al. (1999). One should be aware, however, that these expressions were derived for equidistant time series, and under the 
Table 2. Variability data for the $\gamma$ Doradus stars. Frequencies are in $\mathrm{d}^{-1}$, amplitudes in mmag and phases in degrees. The quoted errors on the frequencies, amplitude ratios and phase differences are standard deviations on the values determined from extensive simulations (see text).

\begin{tabular}{|c|c|c|c|c|c|c|}
\hline ID & $v_{i}$ & $v$ & $A_{B}$ & $S / N_{B}$ & $A_{I} / A_{B}$ & $\phi_{I}-\phi_{B}$ \\
\hline \multirow[t]{3}{*}{ V11 } & $v_{1}$ & $1.165(1)$ & 7.93 & 6.78 & $0.41(4)$ & $-1(5)$ \\
\hline & $v_{2}$ & $1.270(1)$ & 5.12 & 4.37 & $0.40(6)$ & $6(8)$ \\
\hline & $v_{3}$ & $1.400(2)$ & 8.58 & 7.34 & $0.40(3)$ & $3(5)$ \\
\hline V12 & $v_{1}$ & $1.395(4)$ & 9.26 & 5.84 & $0.70(6)$ & $30(6)$ \\
\hline V13 & $v_{1}$ & $0.830(1)$ & 8.10 & 6.00 & $0.39(4)$ & $13(6)$ \\
\hline \multirow[t]{3}{*}{ V14 } & $v_{1}$ & $0.758(4)$ & 2.130 & 4.57 & $0.68(13)$ & $33(12)$ \\
\hline & $v_{2}$ & $0.870(1)$ & 17.750 & 38.08 & $0.43(2)$ & $-8(2)$ \\
\hline & $v_{3}$ & $0.927(1)$ & 4.090 & 8.77 & $0.31(8)$ & $88(18)$ \\
\hline \multirow[t]{3}{*}{ V15 } & $v_{1}$ & $0.495(4)$ & 3.60 & 3.16 & $0.46(13)$ & $35(17)$ \\
\hline & $v_{2}$ & $0.614(1)$ & 14.93 & 13.10 & $0.48(2)$ & $4(2)$ \\
\hline & $v_{3}$ & $1.130(1)$ & 6.28 & 5.51 & $0.45(4)$ & $131(5)$ \\
\hline \multirow[t]{4}{*}{ V16 } & $v_{1}$ & $0.474(20)$ & 9.599 & 5.02 & $0.29(3)$ & $14(13)$ \\
\hline & $v_{2}$ & $0.795(1)$ & 20.603 & 10.78 & $0.52(2)$ & $-13(2)$ \\
\hline & $v_{3}$ & $0.918(3)$ & 11.459 & 6.00 & $0.49(4)$ & 1(4) \\
\hline & $v_{4}$ & $1.730(13)$ & 6.144 & 3.21 & $0.73(11)$ & $-4(8)$ \\
\hline \multirow[t]{6}{*}{ V17 } & $v_{1}$ & $0.136(1)$ & 10.875 & 7.64 & $0.68(4)$ & $21(3)$ \\
\hline & $v_{2}$ & $0.606(1)$ & 28.233 & 19.85 & $0.46(2)$ & $4(2)$ \\
\hline & $v_{3}$ & $0.645(7)$ & 15.658 & 11.01 & $0.51(3)$ & $-11(3)$ \\
\hline & $v_{4}$ & $1.268(1)$ & 7.362 & 5.17 & $0.55(5)$ & $-3(5)$ \\
\hline & $v_{5}$ & $1.537(2)$ & 7.213 & 5.07 & $0.35(11)$ & $28(15)$ \\
\hline & $v_{6}$ & $2.439(1)$ & 5.711 & 4.01 & $0.65(8)$ & $-2(7)$ \\
\hline \multirow[t]{2}{*}{ V18 } & $v_{1}$ & $0.206(1)$ & 8.939 & 9.51 & $0.80(4)$ & $-15(3)$ \\
\hline & $v_{2}$ & $0.674(2)$ & 3.767 & 4.01 & $0.96(11)$ & $-14(6)$ \\
\hline \multirow[t]{5}{*}{ V19 } & $v_{1}$ & $0.156(1)$ & 7.190 & 6.13 & $0.56(4)$ & 1(4) \\
\hline & $v_{2}$ & $0.442(2)$ & 15.774 & 13.44 & $0.51(2)$ & $1(2)$ \\
\hline & $v_{3}$ & $0.583(1)$ & 20.767 & 17.70 & $0.39(2)$ & $0(3)$ \\
\hline & $v_{4}$ & $1.344(3)$ & 6.928 & 5.90 & $0.51(5)$ & $-27(5)$ \\
\hline & $v_{5}$ & $1.593(5)$ & 6.902 & 5.88 & $0.51(6)$ & 5(6) \\
\hline \multirow[t]{3}{*}{ V20 } & $v_{1}$ & $0.842(25)$ & 7.610 & 11.23 & $0.43(5)$ & $-28(7)$ \\
\hline & $v_{2}$ & $0.883(1)$ & 9.963 & 14.70 & $0.66(5)$ & $-2(3)$ \\
\hline & $v_{3}$ & 1.701(16) & 3.011 & 4.44 & $0.47(8)$ & $-28(11)$ \\
\hline \multirow[t]{3}{*}{ V21 } & $v_{1}$ & $0.503(1)$ & 5.069 & 3.50 & $0.53(9)$ & $4(15)$ \\
\hline & $v_{3}$ & $0.648(1)$ & 17.793 & 12.30 & $0.40(2)$ & $-15(2)$ \\
\hline & $v_{3}$ & $1.740(1)$ & 5.434 & 3.76 & $0.60(5)$ & $29(6)$ \\
\hline \multirow[t]{2}{*}{ V22 } & $v_{1}$ & $0.700(1)$ & 14.214 & 6.18 & $0.44(3)$ & $-8(4)$ \\
\hline & $v_{2}$ & $1.378(1)$ & 12.684 & 5.52 & $0.42(3)$ & $2(4)$ \\
\hline \multirow[t]{2}{*}{ V23 } & $v_{1}$ & $1.168(3)$ & 9.616 & 6.35 & $0.47(4)$ & $1(5)$ \\
\hline & $v_{2}$ & $1.260(3)$ & 14.415 & 9.52 & $0.52(3)$ & $12(3)$ \\
\hline V24 & $v_{1}$ & $0.916(1)$ & 14.458 & 12.25 & $0.45(3)$ & $16(5)$ \\
\hline \multirow[t]{2}{*}{$\mathrm{V} 25$} & $v_{1}$ & $1.178(1)$ & 14.747 & 10.84 & $0.60(3)$ & $12(2)$ \\
\hline & $v_{2}$ & $1.987(2)$ & 7.055 & 5.19 & $0.50(5)$ & $-18(8)$ \\
\hline
\end{tabular}

assumption that the true oscillation frequencies are known. Our time series are notoriously non-equidistant. In addition, as many of our targets are multi-periodic, we can expect that the derived frequencies for the lowest-amplitude modes may deviate somewhat from the true eigenfrequencies. Hence, the standard formulas for calculating errors are not directly applicable to our data set.

We therefore used extensive simulations to obtain uncertainty estimates. For each star we simulated $B$ and $I$ lightcurves with exactly the same time sampling as for the observed time series. Each time series consisted of a sum of sines with exactly the same frequencies, amplitudes and phases as derived from the observed time series. Next, white noise was added with the same rms value as in the residuals of the observed time series. These time series were then subjected to an automated analysis procedure that mimicked accurately how we analysed the observed time series. Oscillation frequencies were searched for in the $B$ time series with cyclic prewhitening, and these frequency values were then used to perform a least-squares fit of the $B$ and $I$ time series to obtain the amplitudes and phases. The frequency analysis could be automated because the algorithm searched in predefined intervals $\left[v_{i}-\frac{2}{T}, v_{i}+\frac{2}{T}\right]$ where $v_{i}$ is the $i$ th observed frequency (used as input to construct the synthetic time series) and $T$ is the total time span of the time series. From the result of this fit, amplitude ratios and phase difference could be computed. For each star, the procedure was then repeated 4000 times. The result is for each oscillation mode of each star, a set of 4000 amplitude ratios and a set of 4000 phase differences, one for each noise realisation in the time domain. Note that because of the setup with the predefined intervals, the automated procedure cannot be fooled by one-day aliases. It can still be misled, however, by alias frequencies smaller than $2 / T$.

There are two questions we would like to answer with these simulations. First, can we retrieve the frequencies, amplitudes and phase that were used as input to construct the synthetic time series? This is a necessary condition to have confidence in the observational results, which are listed in Tables 1 and 2. The short answer is "yes". The sets of simulations can be used to construct histograms of the fit parameters, which in turn can be used to compute average values and standard deviations. The a posteriori derived mean frequencies are always very close to the input frequencies, which is reassuring. Sometimes, however, the histogram is bimodal or even trimodal with well separated peaks. For these frequencies the problem of aliasing is very severe, and this should be taken into account when determining the uncertainties.

Second question: how large are the uncertainties of the observed amplitude ratios and phase differences? We use the variances derived from the simulated datasets as an uncertainty measure for the observed quantities. Always the total variance, i.e. intra-variance + inter-variance, was computed taking into account possible confusion with window aliases. The results are shown in Fig. 4 for the $\gamma$ Doradus stars. For every target we checked whether the highest-amplitude mode showed aliasing in the simulated data (i.e. a multi-modal histogram) and designated the ones that don't, with red triangles in the figure. We regard the observational amplitude ratios and phase differences of these latter modes as most reliable. As we expected, all our uncertainty estimates are comparable or somewhat larger than the estimates we would have obtained with the formalism of Breger et al. (1999). We postpone futher discussion of Fig. 4 to Sect. 5.

As a final comment we should mention that for 3 modes $\left(v_{1}\right.$ of V12, $v_{2}$ of V15, and $v_{4}$ of V17) the phase differences showed a weak trend with respect to the frequencies in the dataset of 4000 realisations. This signals that we should treat the observational values for these 3 modes with caution.

\section{Oscillating blue straggler stars}

NGC 2506 has a known population of 12 BS stars, which are all probable cluster members based on proper motions (Xin \& Deng 2005; Chiu \& van Altena 1981). Kim et al. (2001) found 3 oscillating BS stars (V1-V3), of which one (V3), was not included in the proper motion study of Chiu \& van Altena. We discover an additional three oscillating BS stars (V6-V8) of which V7 has a membership probability of $42 \%$ from the proper motion study, while the proper motions of the other two were not measured. Although we do not have spectroscopic data to pinpoint their membership status, all six stars have Strömgren indices in agreement with cluster membership - their $m_{1}$ indices, for instance, are similar to those of the cluster turn-off stars. The positions of the six stars in the CMD are marked with blue 
Table 3. Stellar parameters for the variables in NGC 2506. Type names refer to oscillating blue straggler for BS, detached eclipsing binary for $\mathrm{DEB}$, contact binary for $\mathrm{CB}, \gamma$ Doradus for GD, and eclipsing binary (unspecified) for EB. V10 has not been classified, and Strömgren photometry is not available for V8, V22 and V28.

\begin{tabular}{|c|c|c|c|c|c|c|c|c|c|c|c|c|c|c|c|}
\hline ID & $\alpha_{2000}$ & $\delta_{2000}$ & $V$ & $b-y$ & $v-y$ & $m_{1}$ & $c_{1}$ & $(b-y)_{0}$ & $c_{0}$ & $T_{\text {eff }}$ & $M_{V}$ & $M_{\text {bol }}$ & $B C$ & $\log L$ & Type \\
\hline$\overline{\mathrm{V} 1}$ & 75958.1 & -104554 & 13.660 & 0.150 & 0.468 & 0.168 & 0.994 & 0.096 & 0.983 & 8330 & 0.965 & 0.903 & -0.062 & 1.537 & $\overline{\mathrm{BS}}$ \\
\hline $\mathrm{V} 2$ & 75953.6 & -104548 & 14.510 & 0.185 & 0.548 & 0.178 & 0.819 & 0.131 & 0.808 & 7950 & 1.815 & 1.783 & -0.031 & 1.185 & BS \\
\hline V3 & 75957.9 & -104720 & 14.727 & 0.207 & 0.561 & 0.147 & 0.820 & 0.153 & 0.809 & 7740 & 2.032 & 2.009 & -0.022 & 1.095 & BS \\
\hline V4 & 80008.2 & -104550 & 14.645 & 0.292 & 0.706 & 0.122 & 0.586 & 0.238 & 0.575 & 6920 & 1.950 & 1.900 & -0.050 & 1.138 & DEB \\
\hline V5 & 80010.3 & -104317 & 17.430 & 0.456 & 1.133 & 0.221 & 0.199 & 0.402 & 0.188 & & & & & & DEB \\
\hline V6 & $\begin{array}{llll}8 & 00 & 17.2\end{array}$ & -104535 & 14.677 & 0.243 & 0.701 & 0.215 & 0.651 & 0.189 & 0.640 & 7360 & 1.982 & 1.965 & -0.017 & 1.113 & BS \\
\hline V7 & 75953.4 & -104712 & 14.694 & 0.220 & 0.599 & 0.159 & 0.752 & 0.166 & 0.741 & 7600 & 1.999 & 1.983 & -0.015 & 1.105 & BS \\
\hline V8 & 80034.5 & -104650 & 13.80 & 0.22 & & & & & & & & & & & BS \\
\hline V9 & 80005.4 & -104339 & 15.289 & 0.268 & 0.666 & 0.130 & 0.681 & 0.214 & 0.670 & 7160 & 2.594 & 2.563 & -0.030 & 0.873 & $\mathrm{~EB}$ \\
\hline V10 & 75954.9 & -104646 & 13.079 & 0.522 & 1.287 & 0.243 & 0.513 & 0.468 & 0.502 & & & & & & ? \\
\hline V11 & 80004.6 & -104843 & 15.454 & 0.275 & 0.681 & 0.131 & 0.621 & 0.221 & 0.610 & 7080 & 2.759 & 2.720 & -0.038 & 0.810 & GD \\
\hline V12 & 80005.0 & -104711 & 15.508 & 0.269 & 0.654 & 0.116 & 0.654 & 0.215 & 0.643 & 7140 & 2.813 & 2.780 & -0.032 & 0.786 & GD \\
\hline V13 & 80004.7 & -104639 & 15.599 & 0.269 & 0.663 & 0.125 & 0.621 & 0.215 & 0.610 & 7130 & 2.904 & 2.870 & -0.034 & 0.750 & GD \\
\hline V14 & 75955.8 & -104841 & 15.376 & 0.262 & 0.687 & 0.163 & 0.610 & 0.208 & 0.599 & 7180 & 2.681 & 2.652 & -0.029 & 0.838 & GD \\
\hline V15 & 75956.5 & -104703 & 14.983 & 0.267 & 0.676 & 0.142 & 0.566 & 0.213 & 0.555 & 7120 & 2.288 & 2.253 & -0.034 & 0.997 & GD \\
\hline V16 & 75957.7 & -104609 & 15.333 & 0.243 & 0.624 & 0.138 & 0.668 & 0.189 & 0.657 & 7370 & 2.638 & 2.621 & -0.017 & 0.850 & GD \\
\hline V17 & 80007.4 & -104333 & 15.521 & 0.257 & 0.662 & 0.148 & 0.621 & 0.203 & 0.610 & 7230 & 2.826 & 2.802 & -0.024 & 0.778 & GD \\
\hline V18 & 80001.0 & -104506 & 15.517 & 0.254 & 0.642 & 0.134 & 0.632 & 0.200 & 0.621 & 7260 & 2.822 & 2.800 & -0.021 & 0.778 & GD \\
\hline V19 & 75949.7 & -104635 & 15.050 & 0.261 & 0.662 & 0.140 & 0.581 & 0.207 & 0.570 & 7180 & 2.355 & 2.326 & -0.029 & 0.968 & GD \\
\hline V20 & 80000.8 & -104347 & 15.138 & 0.264 & 0.673 & 0.145 & 0.636 & 0.210 & 0.625 & 7170 & 2.443 & 2.414 & -0.029 & 0.933 & GD \\
\hline V21 & 80001.6 & -104327 & 15.424 & 0.281 & 0.695 & 0.133 & 0.610 & 0.227 & 0.599 & 7020 & 2.729 & 2.685 & -0.044 & 0.824 & GD \\
\hline V22 & $\begin{array}{llll}8 & 00 & 10.3\end{array}$ & -104042 & 15.10 & 0.29 & & & & & & & & & & & GD \\
\hline V23 & 75957.7 & -104255 & 15.246 & 0.269 & 0.685 & 0.147 & 0.591 & 0.215 & 0.580 & 7110 & 2.551 & 2.516 & -0.035 & 0.892 & GD \\
\hline V24 & 80013.3 & -104949 & 15.299 & 0.263 & 0.652 & 0.126 & 0.627 & 0.209 & 0.616 & 7180 & 2.604 & 2.575 & -0.029 & 0.868 & GD \\
\hline V25 & 80005.3 & -105129 & 15.231 & 0.247 & 0.641 & 0.147 & 0.644 & 0.193 & 0.633 & 7320 & 2.536 & 2.517 & -0.019 & 0.892 & GD \\
\hline V26 & 80026.5 & -104839 & 13.058 & 0.632 & 1.631 & 0.367 & 0.314 & 0.578 & 0.303 & & & & & & EB \\
\hline V27 & 80028.2 & -104713 & 17.175 & 0.368 & 0.912 & 0.176 & 0.219 & 0.314 & 0.208 & & & & & & EB \\
\hline V28 & 75953.2 & -105326 & 18.60 & 1.50 & & & & & & & & & & & CB \\
\hline
\end{tabular}

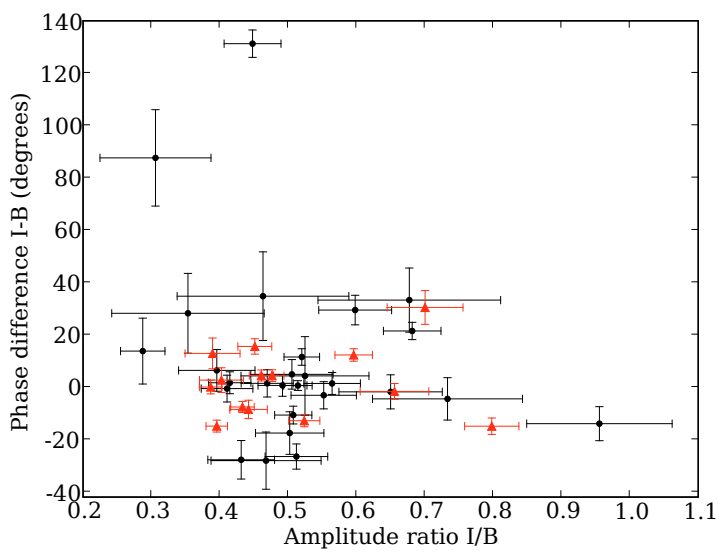

Fig. 4. The amplitude ratio $I / B$ versus the phase difference $I-B$ for all oscillation frequencies detected in the $\gamma$ Doradus stars. The coordinates of the bullets are the observed values, the error bars were computed from the simulated time series. Red triangles are used for those highestamplitude modes for which no aliasing is observed in the simulated time series.

squares in Fig. 3 and the basic parameters are given in Table 3. Furthermore, the six stars have short oscillation periods (Table 1, frequencies around $10-20 \mathrm{~d}^{-1}$ ), are mostly multiperiodic and, except V2, have low amplitudes (mmag). These are the signatures of $\delta$ Scuti type oscillations. Because the $\delta$ Scuti instability strip overlaps the region of the blue stragglers (Fig. 3) the six stars are most likely cluster members, as an instrinsically fainter foreground star, or a brighter background star, would not be expected to display $\delta$ Scuti type oscillations.
Light curves from a single night for all 6 stars are shown in Fig. 5. V6 and V8 are outside the MEROPE FOV so we have only single site data for these stars. This results in a different spectral window function, however for both the single- and the dual-site data, prominent sidelobes are present in the amplitude spectra. We show such spectra of V1-V3 in Fig. 6 and of V6-V8 in Fig. 7. The window function for the stars observed at both sites is best illustrated by V2, which has a single dominant frequency, while that of the single-site data is illustrated by V6. For this star, the signature of the dominant frequency is, however, distorted by a low-amplitude mode at higher frequency. Below, we discuss the individual stars in more detail.

\subsection{The known variables V1-V3}

Kim et al. (2001) detected short-period oscillations in V1-V3, with dominant frequencies of 14.742, 10.854 and $12.264 \mathrm{~d}^{-1}$, respectively, in good agreement with our results. They classified all three variables as $\delta$ Scuti stars, but only V1 as a blue straggler. However, based on our new, more precise CMD (Fig. 3), the six short-period variables are bluer than the cluster turn-off and should therefore all be classified as blue stragglers. Using a period-luminosity relation for $\delta$ Scuti stars, Kim et al. arrived at a tentative identification for the dominant modes of V1 and V2 as 3 rd radial overtone and the fundamental radial mode, respectively.

$\mathrm{V} 1$ is the brightest and bluest star of the variables. The light curves of this star were corrected for low-frequency $\left(0-2 \mathrm{~d}^{-1}\right)$ variations at the level of 2-3 mmag before the data were merged. These variations caused minor mis-matches between the MEROPE and DFOSC data, and must therefore be ascribed 


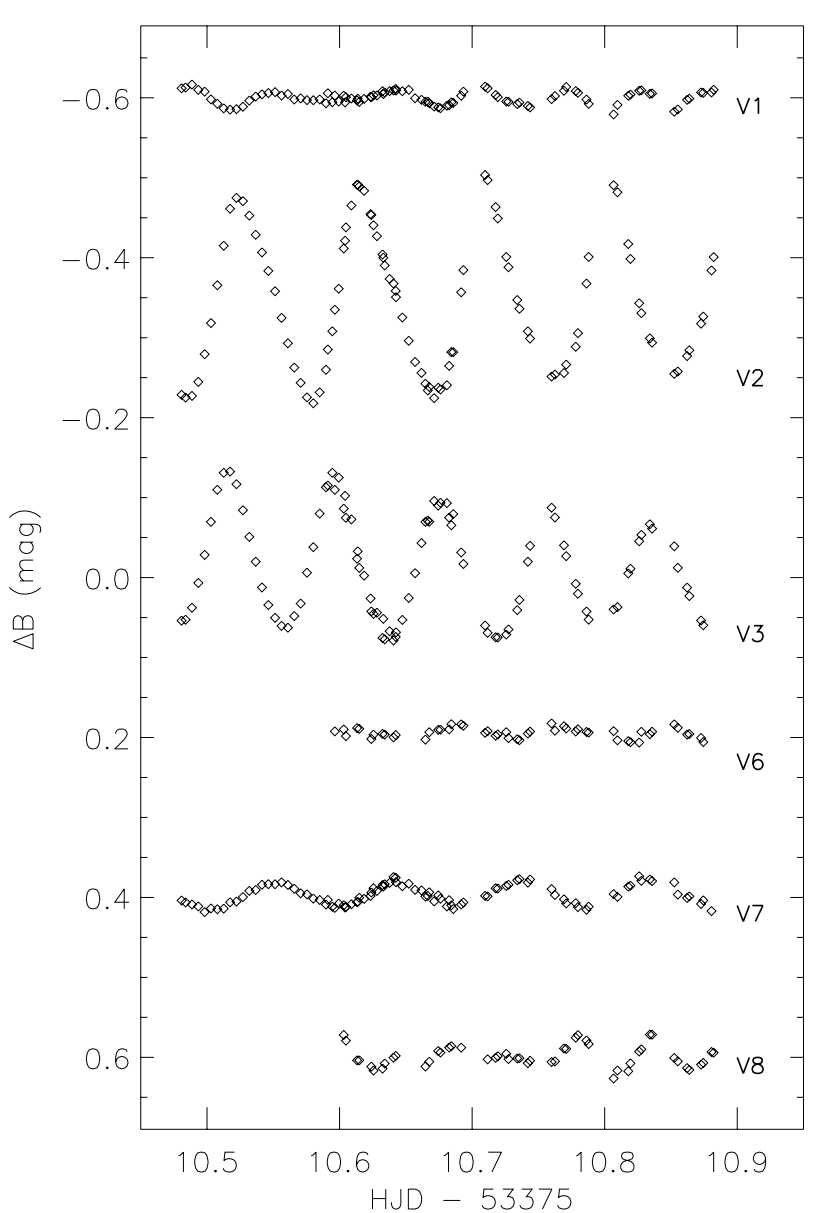

Fig. 5. A single night of data on the BS stars. V6 and V8 were observed from La Silla only.

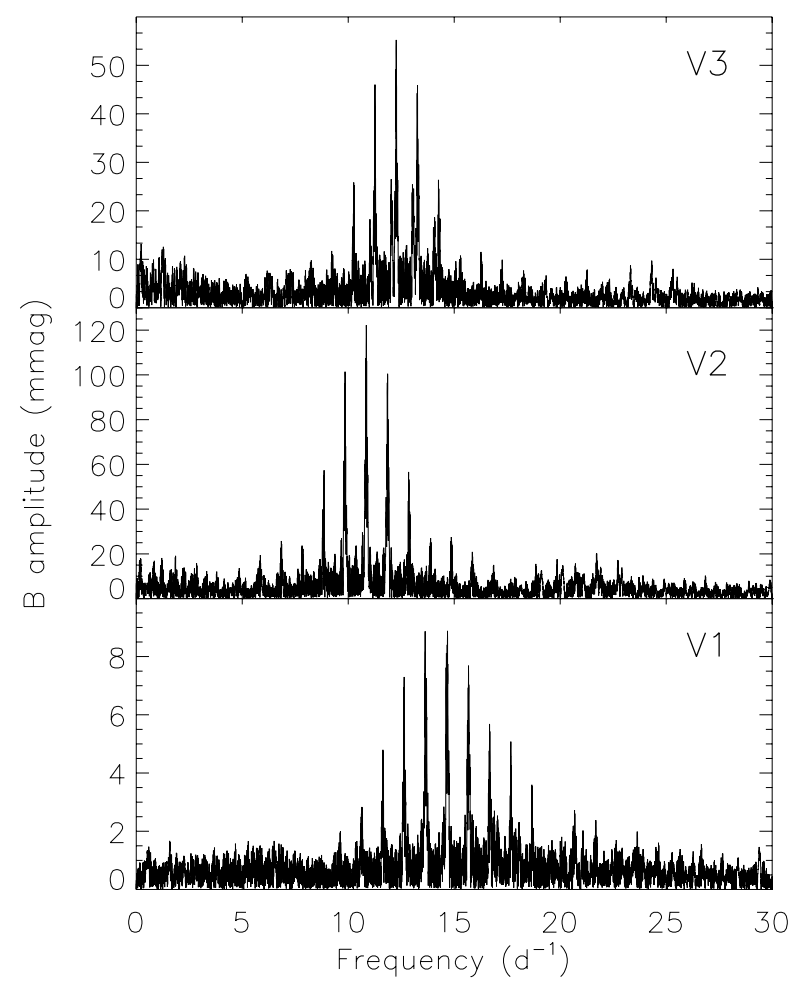

Fig. 6. $B$ amplitude spectra of the three known, oscillating BS stars (V1-V3). Notice the differences in $y$-scale between the panels. to instrumental or atmospheric effects (see Sect. 3.1). In the final light curves, we detect 7 frequencies in the range $13-18 \mathrm{~d}^{-1}$, with amplitudes between 2 and 5 mmag (Table 1). V2 is a highamplitude variable $(0.12 \mathrm{mag}$ in $B)$. The data from the two sites match up well, and no low-frequency filtering was necessary when merging the data. The dominant mode at $10.854 \mathrm{~d}^{-1}$ is distorted as we detect $2 v_{1}$ and $3 v_{1}$ as well. $v_{2}$ appears to be an independent term, while $v_{3}$ is equal to $v_{1}+v_{2}$. Such asymmetries and mode-couplings are common in high-amplitude $\delta$ Scuti stars.

Some of the BS stars fall within the $\gamma$ Doradus instability strip as well. V3 is located in the CMD just to the blue side of the $\gamma$ Doradus instability strip, but it is nevertheless the only one of the six oscillating BS stars which seems to display both low- and high-frequency variability. The fast variations in V3 (Figs. 2, 5 and 6) are clearly visible. We detect 8 frequencies $\left(11-25 \mathrm{~d}^{-1}\right)$, with amplitudes in the range 3-56 mmag in $B$. When merging the data from the two sites, it was necessary first to remove a relatively strong $(25 \mathrm{mmag})$ signal at $1 \mathrm{~d}^{-1}$ from the DFOSC $B$-data only, as mentioned in the caption of Fig. 2. The other three data sets (MEROPE BI, DFOSC $I$ ) remained uncorrected. During the frequency analysis of the merged data, we found that three low-frequency terms were present in both the $B$ - and $I$-data. The $I / B$ amplitude ratios of these frequencies are about 0.4 , which is very similar to amplitude ratios of the $\gamma$ Doradus stars to be discussed below. This points toward oscillations as a possible explanation of the low-frequency variability in V3. This result should be confirmed, but it is an interesting possibility that V3 may oscillate simultaneously in g-modes as the $\gamma$ Doradus stars, and in p-modes as the $\delta$ Scuti stars.

\subsection{The new variables $66-V 8$}

In addition to the three oscillating BS stars discussed above, we detected short-period oscillations in the three stars V6-V8 as well. The amplitude spectra are shown in Fig. 7.

As mentioned above, we only have data from DFOSC on V6. As in the case of $\mathrm{V} 3$, the $B$ data included a relatively strong signal at $1 \mathrm{~d}^{-1}$, which has been subtracted in Fig. 7. We detect three frequencies in V6, although $v_{1}$ does not stand out clearly in the $I$ data. There appears to be further frequencies present in the range $10-15 \mathrm{~d}^{-1}$, but these are below the $4 \sigma$ detection threshold of our data. Although V6 is located inside the $\gamma$ Doradus instability strip as well, we do not find evidence for low-frequency, $\gamma$ Doradus type variability.

For V7, we detect a single frequency, but the residuals after subtracting $v_{1}$ indicate that further frequencies may be present - especially a peak near 9.05 or $10.05 \mathrm{~d}^{-1}$ with a $B$-amplitude of about 2 mmag just escapes a $4 \sigma$ detection in both the $B$ and $I$-data. V8, on the other hand, is multiperiodic with at least 7 frequencies, although $v_{7}$ does not meet the $4 \sigma$ criterion for detection. It is, however, present in both the $B$ - and $I$-filter data and is therefore included in Table 1. Further frequencies are probably present in the light curves of V8, but escapes detection in our data. No low-frequency filtering has been applied to the V8-data shown in Fig. 7.

We finally mention the star V10, which displays variability at the mmag-level with a periodicity near $10 \mathrm{~d}^{-1}$. This star is, however, positioned in the CMD near the giant stars of the cluster and is close to the saturation limit of our data. Little can be said at this stage about the nature of this object, the variability seems $\delta$ Scuti like, so it is probably not a cluster member. 


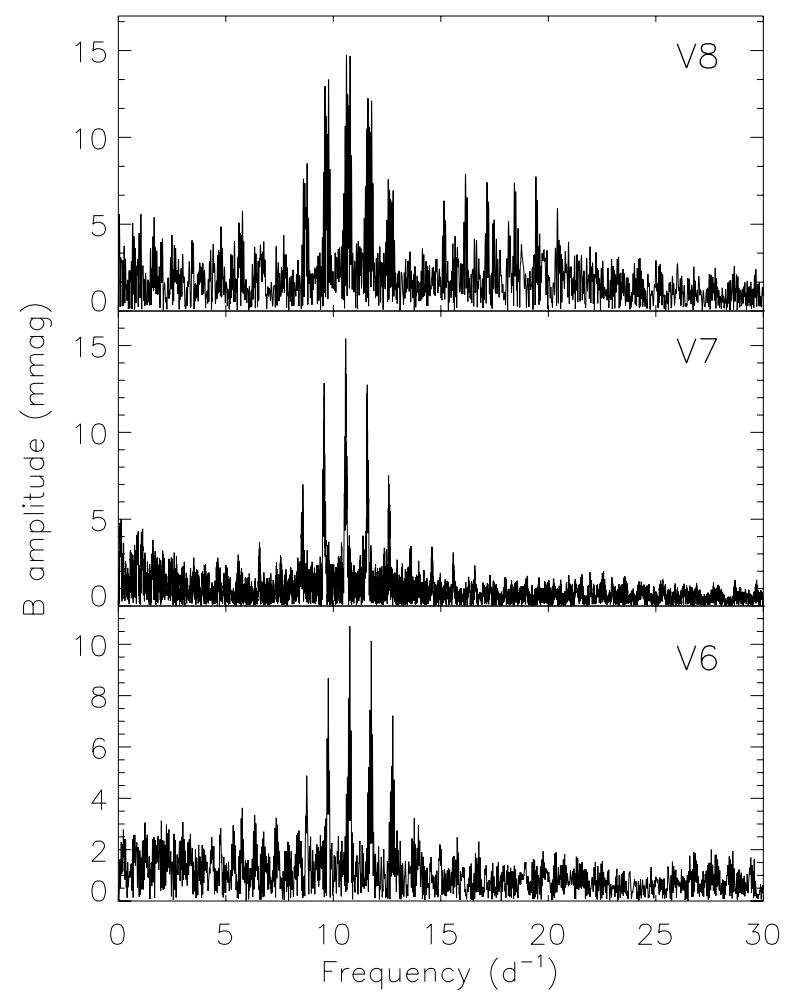

Fig. 7. Amplitude spectra of the new oscillating BS stars V6-V8.

\subsection{Mode-identification of the oscillating BS stars}

Mode-identification in $\delta$ Scuti stars is a notorious problem. Unless the modes can be identified directly, as in the case of the double-mode SX Phe stars, or from combined spectroscopy and photometry for bright stars such as FG Vir (Viskum et al. 1998; Breger et al. 1999; Zima et al. 2006), one must turn to direct comparison with theoretical models. The latter method is further complicated in the present case, as the $\delta$ Scuti variables are blue stragglers, for which the formation history is unknown (see, e.g., Gilliland et al. 1998; Tian et al. 2006). In the case of NGC 2506, cluster parameters and isochrones will be constrained from the binary analysis of spectroscopic (VLT data are already obtained, and will be published in a separate paper) and photometric (this paper) data on detached, eclipsing cluster binaries. In parallel with this, we will initiate dedicated model calculations for the oscillating blue stragglers, but this is beyond the scope of the present paper. Instead, we will use a period-luminosity relation (PLR) from Petersen \& ChristensenDalsgaard (1999, their Eq. (4)), along with expected period ratios for consecutive, radial overtones in $\delta$ Scuti stars, to discuss the detected frequencies (see e.g., Petersen \& ChristensenDalsgaard 1996; Gilliland et al. 1998). Although the period ratios should be evaluated using dedicated models, the ratios are quite constant between models of different mass (e.g., Fig. 21 in Gilliland et al. 1998). One should, however, be cautious when using period ratios for mode-identifications of low-amplitude, multiperiodic $\delta$ Scuti stars, as non-radial modes may easily show the same frequency ratios as the radial overtones, as noted by Poretti (2003). In addition, one should also be cautious of the fact that for the present paper, we are using period ratios from standard models of non-rotating $\delta$ Scuti stars for modeidentification in BS stars. These assumptions should be kept in mind when we in the following discuss the mode-identification of the indvidual BS variables. In Fig. 8 we compare the observed

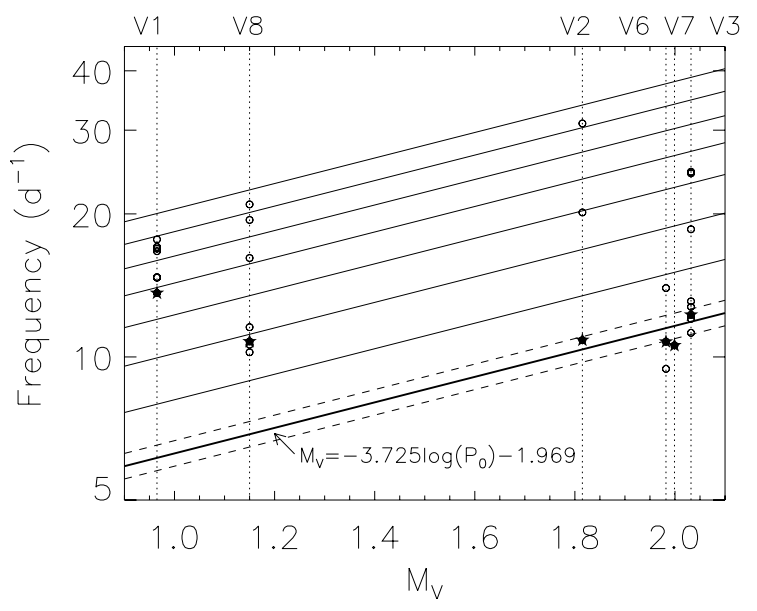

Fig. 8. Comparison between the observed frequencies in the BS stars and a period-luminosity relation for the fundamental mode of $\delta$ Scuti stars (thick solid line) and for consecutive radial overtones (solid lines) based on theoretical frequency ratios from stellar oscillation models. The dashed lines indicate the $1 \sigma$ error on the PLR and the vertical, dotted lines plots the $M_{V}$ of the individual variables. The star-symbols indicate the frequency of highest amplitude in each star.

frequencies with the Period-Luminosity relation for the fundamental (F) modes from Petersen \& Christensen-Dalsgaard (1999) and with consecutive radial overtones.

Kim et al. (2001) identified the dominant frequency of V1 as the third radial overtone (3O), while we find that this frequency $\left(v_{1}\right)$ is better matched with the fourth radial overtone (4O). We note, by the way, that the frequency ratio between $v_{1}$ and $v_{7}$ is 0.771 , which is the expected $\mathrm{F} / 1 \mathrm{O}$ frequency ratio, but such an identification clearly disagrees with the PLR. Instead $v_{7}$ could possibly be $6 \mathrm{O}$, but given the dense frequency spectrum, most, if not all, frequencies are likely non-radial. The dominant frequency in the high-amplitude $\delta$ Scuti star V2 $\left(v_{1}\right)$, on the other hand, is in good agreement with a F-mode classification, as also noted by Kim et al. The frequency $v_{2}$ could be identified as $3 \mathrm{O}$ if we allowed for a $1 \mathrm{~d}^{-1}$ shift to $21.141 \mathrm{~d}^{-1}$. Although we do have significant sidelobes in the amplitude spectra, neither the $B$ or $I$ data support such a shift.

The strongest signal in $\mathrm{V} 3, v_{6}$ (at 56 mmag amplitude in $B$ ), also agrees well with the fundamental mode based on the PLR, while all the other frequencies probably are non-radial. Several frequencies cluster near $12 \mathrm{~d}^{-1}$, and three of them $\left(v_{4}, v_{5}, v_{7}\right)$ form a near equidistant triplet. However, the error simulations puts the largest uncertainty on $v_{7}$, of $0.005 \mathrm{~d}^{-1}$, much smaller than the observed deviation from equidistance of $0.071 \mathrm{~d}^{-1}$. If V3 is a moderate to fast rotator, significant deviations from simple triplets are expected (Kjeldsen et al. 1998), but such conclusions would require comparison with dedicated stellar models as well as a spectroscopically derived rotational velocity.

V6 represents the best case in terms of mode-identification. The frequency of the dominant mode $\left(v_{2}\right)$ agrees with a F-mode classification, and the ratio between $v_{2}$ and $v_{3}$ is 0.771 , which suggests a F/1O identification. $v_{1}$ must then be non-radial. V7 is monoperiodic, but the single frequency is in agreement with the fundamental mode predicted by the PLR, within the uncertainties. We do not have Strömgren indices for V8, but $M_{V} \approx 1.15$ based on the $V$-magnitude. The dense spectrum near $10 \mathrm{~d}^{-1}$ clearly suggests non-radial oscillations, and indeed, we have no evidence for radial modes. 


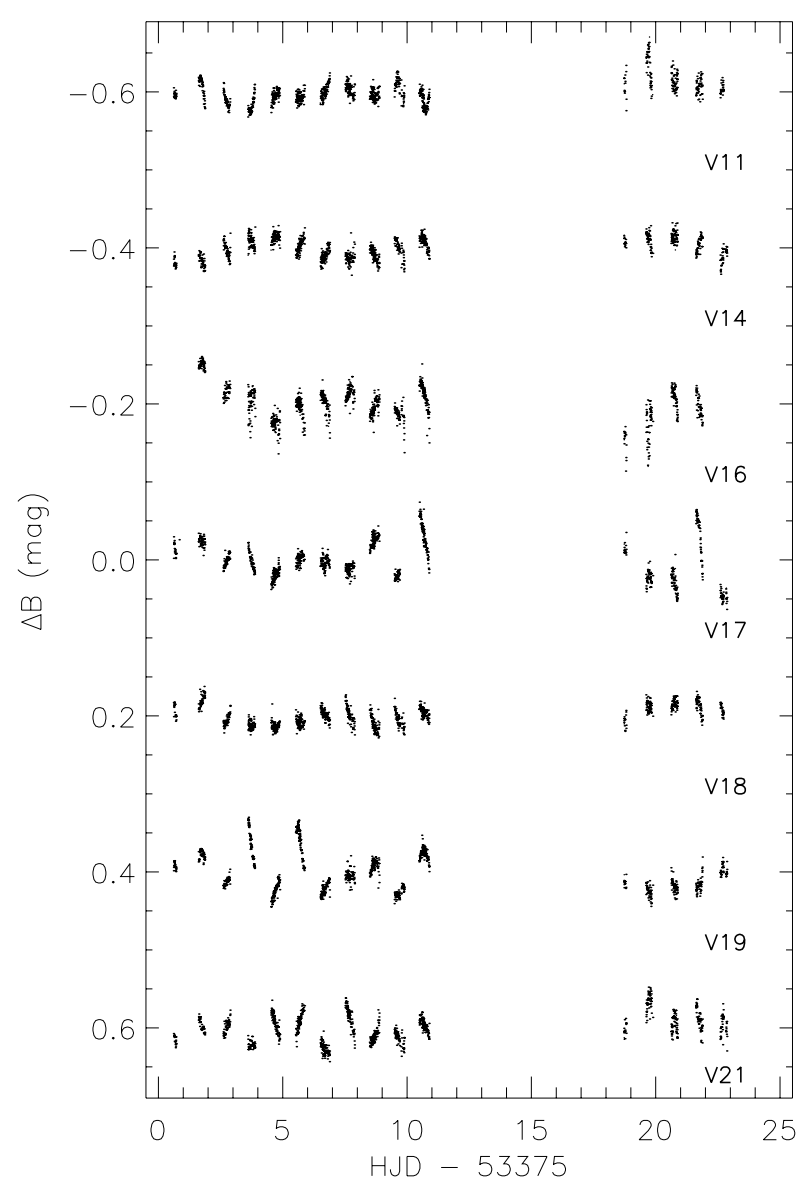

Fig. 9. Light curves for 7 of the long-period variables ( $\gamma$ Doradus) stars in our sample.

To summarize, comparison with the PLR and expected frequency ratios suggest radial modes to be excited in V1 (4O), V2 (F), V3 (F), V6 (F/1O) and V7 (F).

\section{Discovery of $\gamma$ Doradus stars}

Among the new variable stars is a population of 15 stars displaying long-period variability on time scales from hours to days. The amplitudes of variability are in the range 5-30 mmag and several stars are multiperiodic. They are positioned inside the $\gamma$ Doradus instability strip in the CMD (Fig. 3) which, along with the periods and amplitudes suggest that they may be $\gamma$ Doradus stars (Handler 1999; Handler \& Shobbrook 2002; Henry et al. 2005). We will in the following argue that these stars, labeled V11-V25, can in fact be classified as $\gamma$ Doradus stars based on the evidence presented below.

\subsection{Evidence for multiperiodic variability}

In Fig. 9 we show light curves for 7 of the $15 \gamma$ Doradus candidates. They clearly display variability on time scales of days, and beating effects resulting from multiperiodicity is evidently present in several stars. The amplitude spectra of two of the stars are shown in Fig. 10 (V17 and V21), along with the spectral window function shown at the same frequency scale. By comparing to the spectral window, which shows the signature of a single frequency in the amplitude spectrum, it is again evident that several frequencies are present, especially in V17. Indeed,

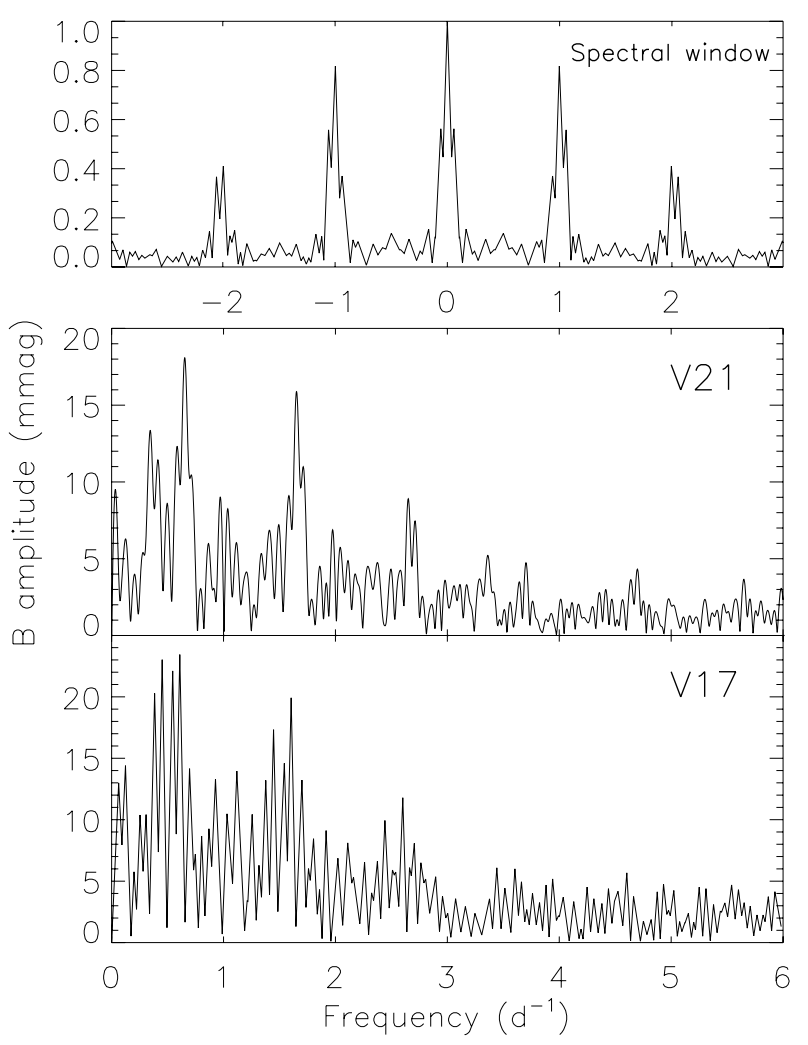

Fig. 10. Spectral window function and examples of amplitude spectra for two stars for which we detect long-period variability.

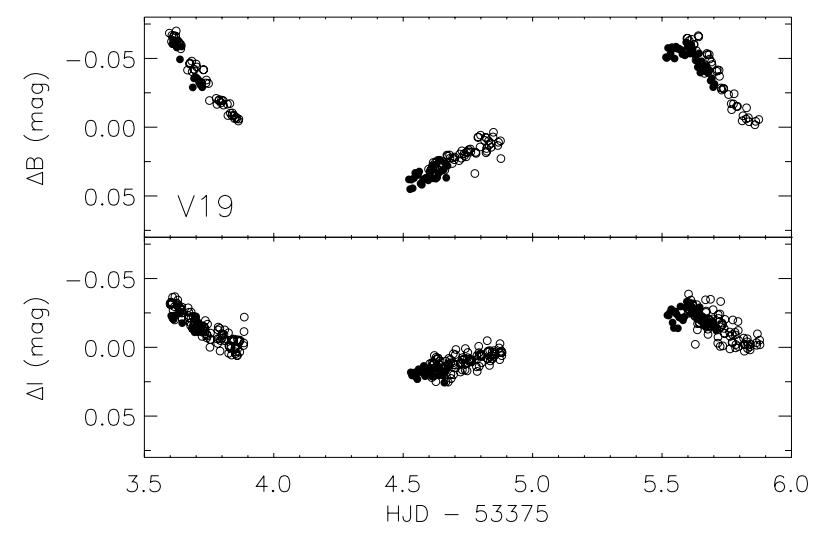

Fig. 11. Three nights of merged data for the $\gamma$ Doradus star V19. Filled circles are data from La Palma, open circles from La Silla. The amplitude difference between $B$ and $I$ is evident.

we detect 6 and 3 frequencies for V17 and V21, respectively, in the range $0.1-2.5 \mathrm{~d}^{-1}$.

In Fig. 11 we show an example of the merging of data from La Palma and La Silla for the variable V19. For 12 of the 15 stars we have data from both sites and in all cases the overlapping data agree well (if this was not the case, the star would be rejected from the list of variables). This excludes an instrumental origin of the long-period variability, which must be intrinsic to the stars. For V22, V24 and V25 we have data from La Silla only, but the variability is in all cases clearly seen, and present in both the $B$ - and $I$-filter data, leaving little room for doubt about the intrinsic nature of the light variability.

All detected frequencies are listed in Table 2. We find 3-6 frequencies in several of the stars, and only three stars appear mono-periodic in our data. In general we find $I / B$ amplitude 
ratios of $0.4-0.6\left(A_{I} / A_{B}\right)$ while a wide range in phase differences is observed. The typical residual noise level in the amplitude spectra in the frequency range $0-6 \mathrm{~d}^{-1}$ are $0.5-2.0 \mathrm{mmag}$ for these $V=15-16$ stars. These numbers explain why Kim et al. (2001) could not find $\gamma$ Doradus stars in their dataset. Indeed, their dataset consisted of 304 images, and they quote a detection limit of $10 \mathrm{mmag}$ at $V=14$. The corresponding number for our dataset is $2 \mathrm{mmag}$, as residual noise levels in the amplitude spectra of the $V=14$ stars in our sample are typically below 0.5 mmag.

\subsection{Are they $\gamma$ Doradus stars?}

To be considered a bona fide $\gamma$ Doradus star, a star should be located in the CMD inside the $\gamma$ Doradus instability strip, be variable on time scales from $\sim 0.4-3$ days, and all mechanisms for variability other than oscillations must be excluded. Such other mechanisms would typically be binarity (ellipsoidal variability) or rotational modulation (star spots).

All 15 stars are positioned inside the $\gamma$ Doradus instability strip near the cluster main sequence turn-off. From isochrone fitting we find that the bluemost point on the cluster sequence corresponds to a mass of $1.4-1.5 M_{\odot}$. This result is corroborated by a preliminary analysis of the binary $\mathrm{V} 4$, which places the primary at the top of the cluster main sequence with a mass of $1.5 \pm 0.1 M_{\odot}$ and the secondary further down the main sequence, below the instability strip, with a mass of $1.2 \pm 0.1 M_{\odot}$ (see Fig. 16). This indicates that the turn-off stars are of early $F$-type ${ }^{1}$, in good agreement with the $\gamma$ Doradus phenomenon occurring in stars of type $A 7-F 5$ (Kaye et al. 1999). The position of the 15 variables near the cluster turn-off is thus fully in agreement with a $\gamma$ Doradus classification.

For the multiperiodic variables in our sample, mechanisms other than oscillations can readily be excluded as the cause of variability (see Handler 1999; Handler \& Shobbrook 2002, for a detailed discussion). In short, ellipsoidal variables and rotationally modulated stars would be characterized by a single frequency, or by two frequencies with a harmonic relation. In the case of spots, two close frequencies may appear. For ellipsoidal variables, amplitude ratios between different passbands are expected to be unity, while $V / B$ amplitude ratios of $0.88-0.89$ are expected for starspot variables (Henry et al. 2005). For oscillations, this ratio is expected to be $0.67-0.80$ (Garrido 2000) and typically $0.3-0.6$ for $I / B$ (see Sect. 5.2), which is also what we find for most frequencies (Table 2).

Among the $15 \gamma$ Doradus stars, there are three mono-periodic stars, but for both V13 and V24, further frequencies are likely present and only just escape detection in our data set. For V13 and V24, the amplitude ratios are also near 0.4 , pointing towards oscillations and clearly rejecting ellipsoidal variability. The near detection of further frequencies, along with the low $I / B$ amplitude ratios suggest that also V13 and V24 are oscillating. For $\mathrm{V} 12$, the amplitude ratio is 0.7 which rejects ellipsoidal variability. V13 (along with V12) are in the Mercator field and the data from March and April show that the variability is coherent over time scales of many months. Star spots are not expected in $\gamma$ Doradus stars due to the early F-type classification (Kaye et al. 1999; Henry et al. 2005), except if they are chemically peculiar. As we will show below, none of the three monoperiodic stars appear chemically different from the rest of the group based on the Strömgren photometry, but all 15 stars have low values of $\delta c_{1}$, which is an indicator of chemical peculiarity

\footnotetext{
1 Allen's Astrophysical Quantities, 4th edition, Arthur N. Cox, Ed.
}
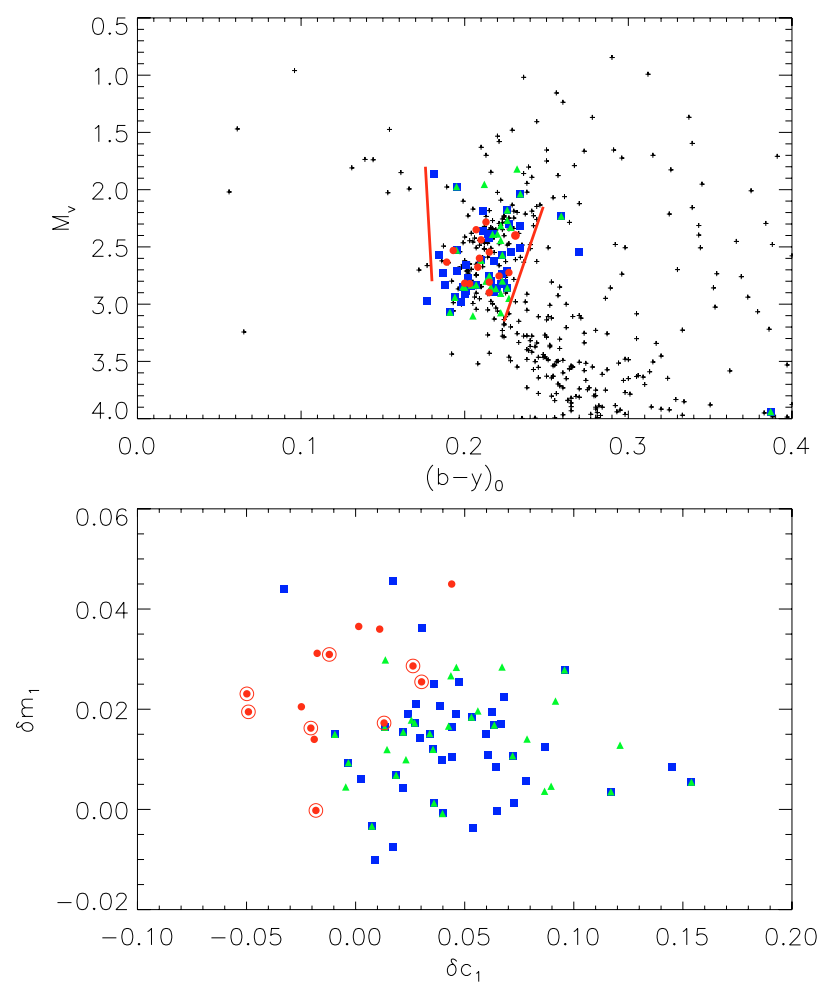

Fig. 12. Comparison of stellar parameters between the NGC $2506 \gamma$ Doradus stars and the known variables (Handler, private communication). The bona fide $\gamma$ Doradus stars are shown as squares, the prime candidates as triangles, the NGC 2506 stars as filled circles, and the instability strip is from Handler \& Shobbrook (2002). In the bottom panel, the multiperiodic (3 or more frequencies) NGC 2506 stars have been marked with an open circle as well.

(Joshi et al. 2006). Thus, we cannot completely rule out that V12 could be a rotationally modulated Ap star, this can only be done spectroscopically. For V22, for which we do not have Strömgren indices except for the position in the CMD inside the instability strip, $v_{1}$ and $v_{2}$ are quite close to be harmonically related, but the $I / B$ amplitude ratios of 0.4 excludes ellipsoidal variability. The evidence therefore suggest oscillations as the cause of variability for 14 of the 15 stars, in agreement with a $\gamma$ Doradus classification.

\subsection{Comparison with the known $\gamma$ Doradus stars}

We have compared the Strömgren indices of the 15 NGC 2506 variables to those of the bona fide and primecandidate $\gamma$ Doradus stars based on updated tables kindly provided by G. Handler. For the latter stars we extracted Strömgren data from the catalogue of E. H. Olsen (private communucation) and used the $b-y$ calibration of Crawford (1975) to derive $\delta c_{1}$ and $\delta m_{1}$. The result is shown in Fig. 12.

In the $M_{V},(b-y)_{0}$ diagram, the NGC 2506 stars overlap completely with the known variables. In the $\left(\delta c_{1}, \delta m_{1}\right)$ diagram, the NGC 2506 stars have a slightly more negative $\delta c_{1}$ and, in general, slightly higher $\delta m_{1}$. The higher $\delta m_{1}$ values suggest lower metallicity, in agreement with the metal content of the cluster, while the more negative $\delta c_{1}$ suggest that the stars may be more chemically peculiar and therefore could be Ap stars (Joshi et al. 2006). The NGC 2506 stars are, however, still located very close to the known $\gamma$ Doradus stars in this $\left(\delta c_{1}, \delta m_{1}\right)$ diagram, where we have marked the variables with three or more frequencies 
with an open circle as well. The variability of those multiperiodic stars are certainly due to oscillations, and this subset includes the two stars with the most negative values of $\delta c_{1}$. The 15 NGC 2506 stars all have very similar indices; they form a homogeneous group in both the CMD and the $\left(\delta c_{1}, \delta m_{1}\right)$ diagram, and nothing suggests that any one star is chemically different from the others. We mention in passing that this supports oscillations as the cause of variability in V12 as well, as the star has indices similar to those of the 14 other $\gamma$ Doradus candidates. The comparison with the known oscillators therefore strengthen the $\gamma$ Doradus classification of the NGC 2506 stars. The minor shift of the NGC 2506 stars in $\delta c_{1}, \delta m_{1}$ may be due to a small zero-point shift of our Strömgren photometry compared to the known variables, which are typically closer field-stars, or to an actual increase in the $\delta c_{1}, \delta m_{1}$ parameter space of the $\gamma$ Doradus stars in general.

The fact that all the NGC $2506 \gamma$ Doradus stars lie on or near the cluster main sequence inside the $\gamma$ Doradus instability strip is a strong indication that they are all cluster members. In fact, plots of various combinations of the Strömgren indices given in Table 3, all suggest that the $\gamma$ Doradus stars are cluster member turn-off stars, in full agreement with the CMD in Fig. 3. If some of the 15 stars were foreground or background objects, and thus were of a different spectral type, they would have Strömgren indices separating them out from the large group of cluster turnoff stars. It would also be difficult to explain the pulsational behaviour of such objects; background Cepheids or RR Lyrae stars would have a characteristic light curve with a larger amplitude of variability. A reddened, background SPB variable could show the same type of variability as in the NGC 2506 stars, but would be unlikely to show Strömgren indices identical to those of Fstars in a metal-poor cluster.

Furthermore, we have VLT/FLAMES spectra for V20, V21 and V24, of which the first two are multiperiodic oscillators, in a data set of 120 stars in the cluster. We have derived preliminary radial velocities based on these spectra (see Sect. 6), and the result is that at least V20 and V24 are cluster members as their mean radial velocity corresponds to the cluster mean velocity. This provides additional, strong support for the classification of all 15 stars as cluster member $\gamma$ Doradus stars. The velocities of V21 are in agreement with cluster membership but long-term $\mathrm{RV}$ variations may be present.

We therefore suggest that the NGC 2506 variables V11-V25 except V12 should be included in the list of bona fide $\gamma$ Doradus stars. V12 should be regarded as a prime candidate until the nature of the variability has been established with certainty. This population of $\gamma$ Doradus stars makes NGC 2506 extremely interesting for studying the $\gamma$ Doradus phenomenon, as these stars cover a large fraction of the instability strip and at the same time are located very near to the evolutionary point of turn-off from the main sequence.

We finally note that we did not find $\delta$ Scuti oscillations in any of the $\gamma$ Doradus stars, despite the fact that many of them are inside the $\delta$ Scuti instability strip as well. This supports the idea that $\gamma$ Doradus and $\delta$ Scuti oscillations are rarely present in the same star (Handler \& Shobbrook 2002; Henry et al. 2005). Both types of oscillation have, however, been found in HD8801 (Henry \& Fekel 2005) and in BD+184914 (Rowe et al. 2007) - and possibly in V3 in this paper. It is interesting to note that we do not find $\delta$ Scuti oscillations in any of the turn-off stars, despite many candidates inside the $\delta$ Scuti instability strip. In contrast, the open cluster NGC 1817 also has turn-off inside (in the middle of) the $\delta$ Scuti instability strip, but that cluster, which

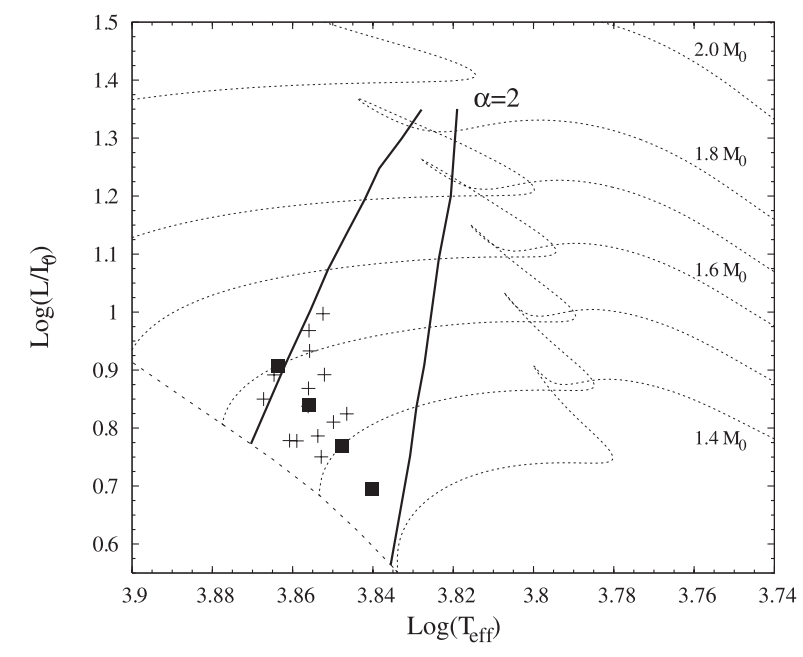

Fig. 13. An HR-diagram showing the 4 generic $\gamma$ Doradus models as squares together with the positions of the $\gamma$ Doradus candidates as crosses. Also shown are the ZAMS, evolutionary tracks and the blue and red border of the $\gamma$ Doradus instability strip for $\ell=1$ modes and for the mixing length parameter $\alpha=2$.

is half as old as NGC 2506, is hosting a population of at least 12 turn-off $\delta$ Scuti stars (Arentoft et al. 2005).

\subsection{Comparison with theoretical predictions}

As mentioned in the introduction, $\gamma$ Doradus stars are rich stellar laboratories to study astrophysical phenomena. This, however, makes them complex and challenging targets for which we need as many additional observational constraints as possible. Such constraints will be obtained when we determine with high precision the masses and radii of the two eclipsing binaries in NGC 2506. The high-resolution spectroscopic datasets involved, obtained with the VLT at Paranal Observatory, are currently being reduced and analysed. The datasets will pinpoint the cluster isochrone with an unprecedented precision for NGC 2506, which will lead to masses, radii and the age of all stars along the cluster main sequence, including the $\gamma$ Doradus stars. In addition, a detailed abundance analysis of cluster stars based on VLT/FLAMES spectra will shed light on the metallicity of the cluster stars. These results, which will be published in a separate paper, will provide the needed, additional constraints and we therefore postpone a detailed seismic analysis of the $\gamma$ Doradus candidates until they are available.

We did, however, perform a preliminary theoretical modeling to verify whether there is at least a crude correspondence between the observed amplitude ratios and phase differences and the theoretical ones. Rather than modeling each target individually and being subjected to the possible large, systematic uncertainties on their positions in the HR-diagram, we choose to compute 4 generic stellar models using the stellar evolution code CLéS (R. Scuflaire, University of Liège, private communication). Each of these models was computed for the same cluster age as specified in Sect. 2 but with different effective temperatures going from the blue to the red border of the $\gamma$ Doradus instability strip. These generic models, together with the positions of the $\gamma$ Doradus candidates are shown in the HR-diagram in Fig. 13.

Consequently, we computed the $\ell=1,2$ and 3 eigenfrequency spectra for each of the generic models using the non-adiabatic oscillation code MAD. This code uses 


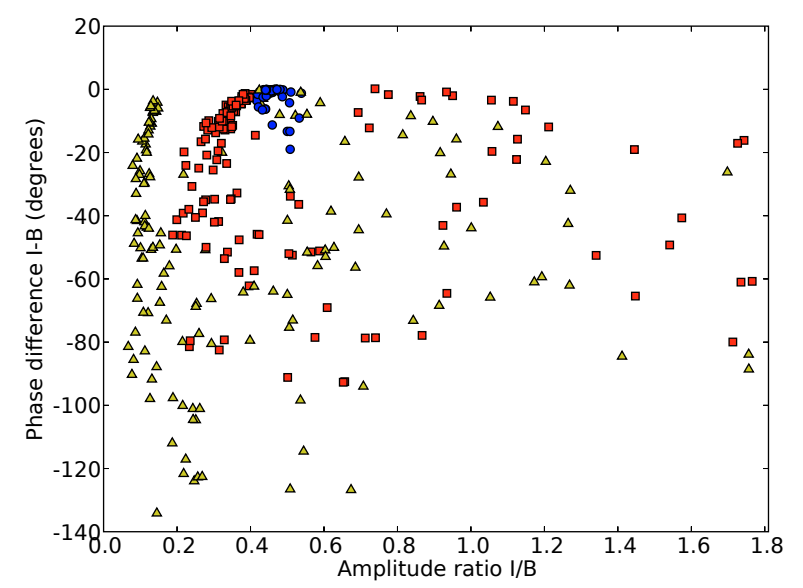

Fig. 14. The theoretical photometric amplitude ratios $I / B$ versus phase difference $I-B$. It concerns the modes of the 4 generic models shown in Fig. 13 for which the eigenfrequency is close to one of the observed frequencies in Table 2 . The blue circles denote $\ell=1$ modes, the red squares $\ell=2$ modes and the yellow triangles $\ell=3$ modes. The results were computed for the cluster metallicity stated in Sect. 2, and for the mixing length parameter $\alpha=2$.

time-dependent convection (TDC) theory as described by Grigahcène et al. (2005) and Dupret et al. (2005a), and was successfully applied to five $\gamma$ Doradus field stars by Dupret et al. (2005b). From these spectra, and for each degree $\ell$, we selected the modes with eigenfrequencies closest to the observed frequencies and computed the non-adiabatic relative amplitude and phase of the $T_{\text {eff }}$ and gravity variation. These quantities were then used to compute the photometric amplitude ratio $I / B$ and phase difference $I-B$. The theoretical model used is the same as the one used in Dupret et al. (2005b), and incorporates Kurucz (1993) atmosphere models and the 4-coefficient non-linear limbdarkening law of Claret (2000). Through interpolation in metallicity grids, the results were computed for the cluster metallicity mentioned in Sect. $2([\mathrm{Fe} / \mathrm{H}]=-0.20)$.

Three important results come out from our comparison between theoretical predictions and observations. First, the generic models are unable to explain the observed oscillation frequencies below $0.25 \mathrm{~d}^{-1}$. In particular, $v_{1}=0.136 \mathrm{~d}^{-1}$ of $\mathrm{V} 17, v_{1}=$ $0.206 \mathrm{~d}^{-1}$ of $\mathrm{V} 18$, and $v_{1}=0.156 \mathrm{~d}^{-1}$ of $\mathrm{V} 19$ are predicted to be stable (i.e. not excited). The most important parameter determining the (in)stability of eigenmodes in $\gamma$ Doradus stars is the mixing-length parameter $\alpha$. We used the value $\alpha=2.0$ because a) testing revealed that this value leads to the largest number of unstable modes, and b) Dupret et al. (2005b) successfully used this value to explain best the observations of five field $\gamma$ Doradus stars. But in the case of our cluster targets this value seems not able to explain the complete observed frequency spectrum. We remark, however, that the observed frequency $v_{3}=0.343 \mathrm{~d}^{-1}$ of the well known $\gamma$ Doradus star 9 Aur is also predicted to be stable by Dupret et al. (2005b).

The second result concerns the photometric phase differences $I-B$. Figure 14 shows the theoretically predicted photometric amplitude ratios $I / B$ and corresponding phase difference $I-B$ for all modes of all targets, assuming degrees $\ell=1,2$ and 3. Comparing this figure with the corresponding observational Fig. 4 reveals a striking difference: for many of the oscillation modes we observe a positive phase difference $I-B$ which cannot be explained by the models. The phase difference of these modes is usually positive well within the error bars, and this is even so for several of the highest-amplitude modes for which no aliasing-problems was detected (red triangles). There is little to compare to in the literature but we mention the relevant information in the forthcoming paper of Cuypers et al. (2007, in preparation). In this paper a sample of $19 \gamma$ Doradus field stars is presented that was photometrically monitored in the Geneva passbands. The authors always find a phase difference between the $U$ and $G$ filter that is very close to zero (within the standard errors). This turns out to be in agreement with the theory. Indeed, we performed similar simulations for the Geneva $U$ and $G$ filters, and we found that the phase difference $U-G$ behaves somewhat different than $I-B$. First, it can theoretically be both positive and negative. Secondly, regardless the degree, the phase difference $U-G$ is almost always close to zero. The results of Cuypers et al. are therefore not surprising. It does seem to show, however, that the phase difference $I-B$ is more suitable for mode identification as it has more discriminating power.

A third remark worth mentioning is the scatter of the observed modes in Fig. 4. Intuitively we would expect more $\ell=1$ modes to be present among our sample as a selection effect because these modes suffer less surface cancellation. This effect seems to be present in the sample of Dupret et al. (2005b) who identified 8 out of 12 studied $\gamma$ Doradus modes unambiguously as dipole modes. Note, however, that the $\ell=1$ modes in Fig. 14 (blue circles) occupy a remarkably small region given the fact that the 4 generic models from which they were computed are spread over a significant part of the $\gamma$ Doradus instability strip. The rather large scatter in the observational diagram in Fig. 4 therefore shows that the majority of our sample of $\gamma$ Doradus modes cannot easily be identified as dipole modes.

Given the evidence, we should conclude that either the observational uncertainties on the photometric amplitude ratios and phase differences are significantly larger than we estimated, or the theoretical model is inadequate. We verified that changing the metallicity (e.g. to $[\mathrm{Fe} / \mathrm{H}]=0.0$ or $[\mathrm{Fe} / \mathrm{H}]=-0.3$ ) does not change qualitatively our results. In our forthcoming theoretical study we will explore a larger parameter space where we will examine in more detail the influence of the overshoot parameter, and the rotational velocity. The latter in particular may be important for $\gamma$ Doradus stars as for these stars the rotation over oscillation frequency ratio can easily be comparable to or larger than 1.

\section{Eclipsing binaries}

As mentioned above, NGC 2506 hosts the two detached, eclipsing binaries V4 and V5. We have used the present photometric data set to determine, for the first time, the periods of these two binaries: 2.868 and 10.078 days for V4 and V5, respectively. The phased light curves are shown in Fig. 15, including some nights of poor quality. The $B$ light-curve of V4 display minor offsets between data taken at the same phase, but on different nights. The same is the case for $I$, although to a lesser extent. Figure 16 illustrates this in more detail. If we assume that the binary is a cluster member, as indicated by the spectroscopic data to be discussed below, the primary component should lie on the cluster sequence near turn-off, i.e., somewhere along the uppermost of the green curves in Fig. 16, lower panel. If we also require the secondary to be on the cluster sequence, and that the combined light of the two main sequence stars should be equal to the observed, total light, we obtain a photometric solution that puts the primary inside the $\gamma$ Doradus instability strip and the secondary somewhat below it, as indicated by the red dots in the diagram. In the upper panel of Fig. 16 we show the light curve in more detail. Additional variability is present on a time scale of several days 


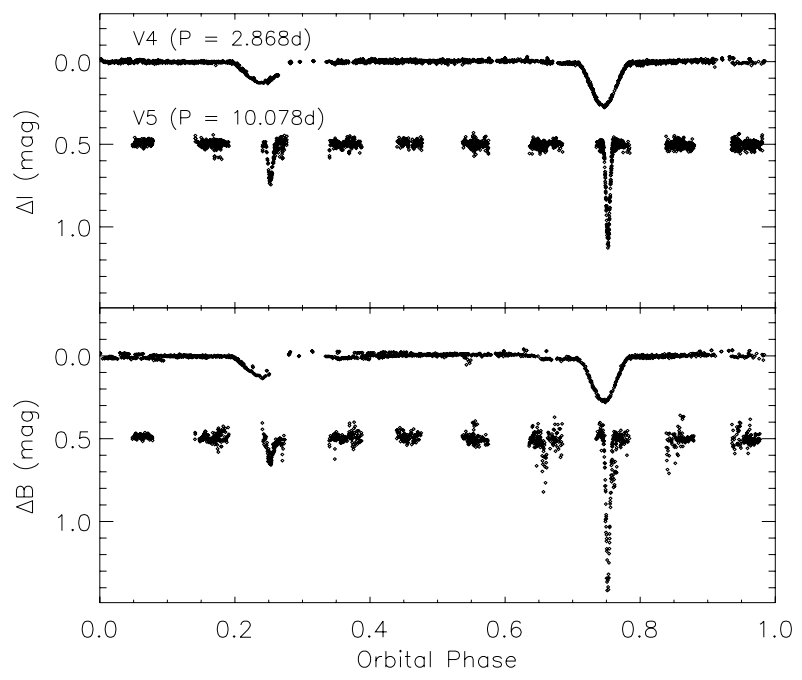

Fig. 15. Phased light curves for the detached binaries V4 and V5.
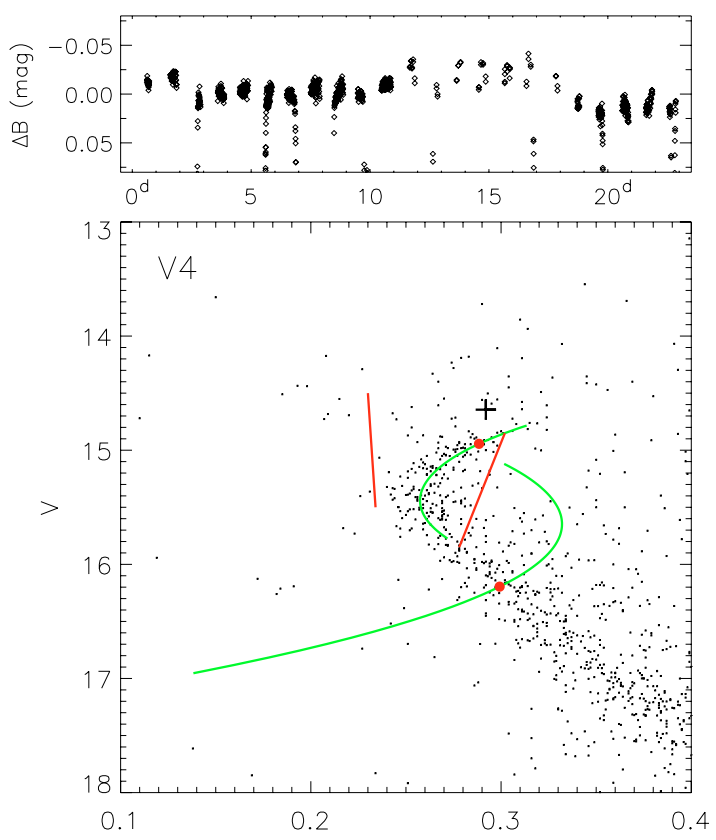

Fig. 16. 24-day light curve (top) and photometric solutions to the V4 binary system. The solid red lines are the $\gamma$ Dor instability strip. The cross denotes the position of V4 in the CMD and the green curves are possible photometric solutions of the two components. The red dots mark a solution that puts both components on the cluster sequence.

which is, however, longer than the typical $\gamma$ Doradus type variability. Further discussion of this object is therefore postponed until all data, including the spectroscopy, are available.

From the photometric data, we also detect four new eclipsing binaries (V9 and V26-V28). Light curves are shown in Figs. 17, 18 and 19 and we refer to Table 3 for photometric indices. V26 is a giant star, near the limit of saturation in our data, especially in $I$. Figure 17 plots the evidence of binarity. V27 (Fig. 18) is possibly a new, detached eclipsing binary positioned in the CMD on the cluster main sequence. A single, probable eclipse was observed in both $B$ and $I$ during one night. Finally, we detect the two binaries V9 and V28 (Fig. 19). V9 is located in the CMD inside the $\gamma$ Doradus instability strip, near the cluster sequence, and may be an Algol- or $\beta$ Lyrae type binary. V28 is faint, red and, from the position in the CMD far from the cluster main sequence, not a cluster member. The short

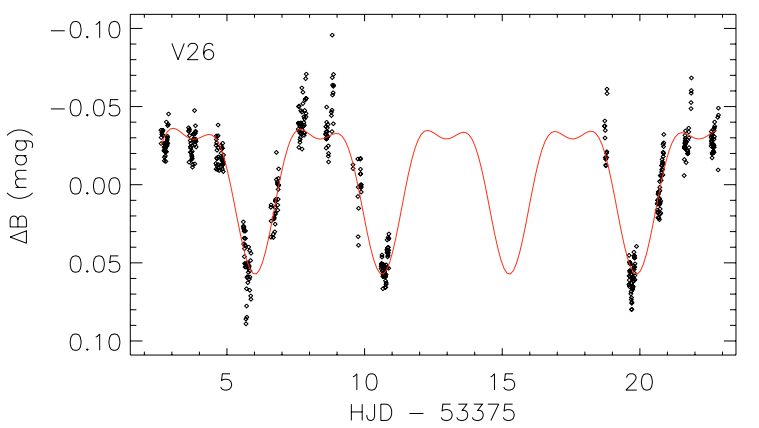

Fig. 17. The new probable binary V26. The red curve shows a 2 -frequency fit with a 1:2 relationship between them.

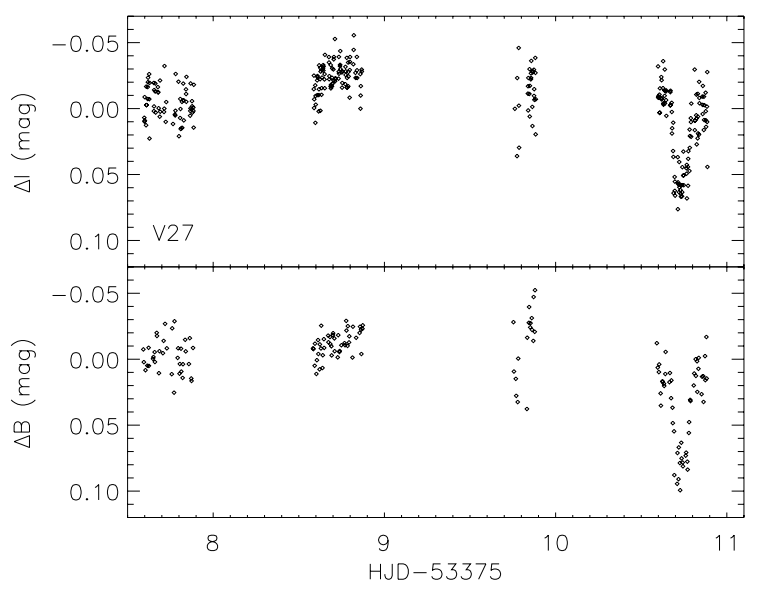

Fig. 18. The new probable binary V27; four nights of data are shown, with a possible eclipse taking place during the last night.

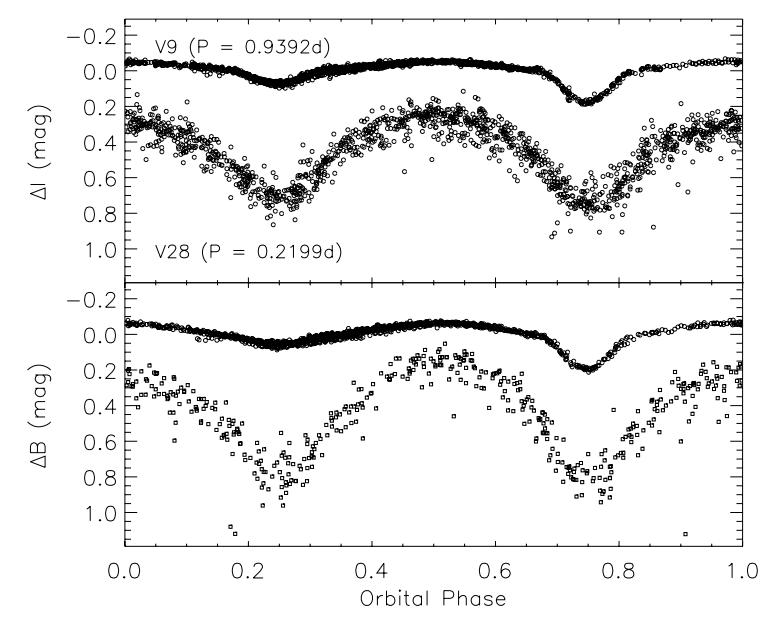

Fig. 19. Phased light curves for the new binaries V9 and V28. The latter object is not a cluster member based on the position in the CMD.

period (0.2199 days), and the light-curve shape, suggest a W UMa classification.

The spectroscopic data-set mentioned above consists of 15 epochs of VLT UVES spectra for 5 stars (V4 and four giants) and Giraffe/FLAMES spectra of 120 stars (including V5) observed in service mode. The aims of these data are to analyse the binary stars for deriving precise, absolute parameters, as well as to use the Giraffe spectra to determine metallicity/abundances, ages and cluster membership. The latter allows a cleaning of the bright part of the CMD for non-members, which in turn allows much stronger constraints to be put on the isochrones. 


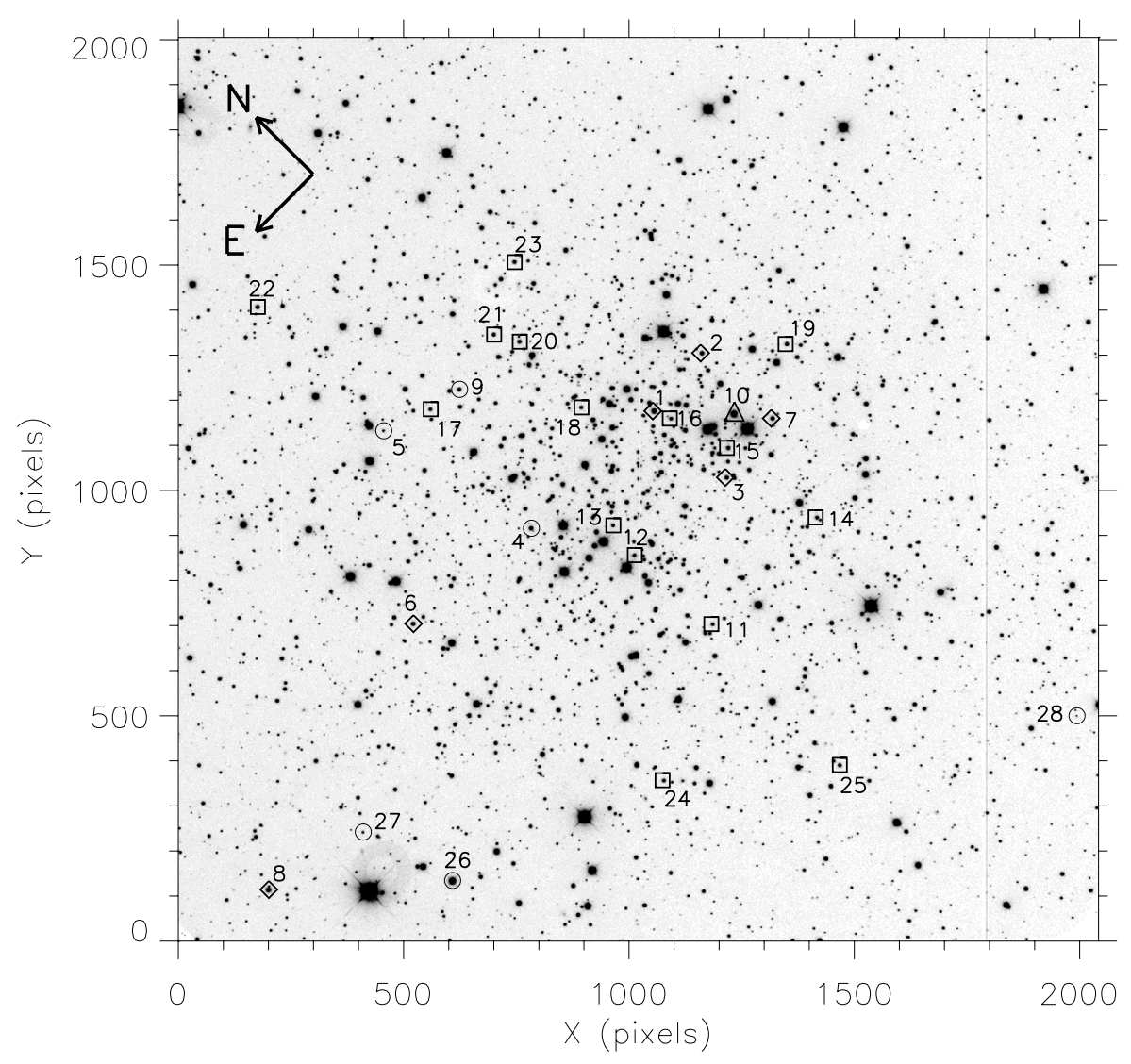

Fig. 20. DFOSC image serving as a finding chart for NGC 2506, with the variables indicated. The image orientation is indicated by arrows.

The analysis of the NGC 2506 spectra is still ongoing and will be the subject of a later paper, but we mention that a preliminary analysis of the UVES spectra shows that V4 is a double-lined binary and a cluster member.

We have also done a preliminary reduction of the Giraffe spectra to derive approximate radial velocities for the $120 \mathrm{ob}-$ served stars, among these V5 and V9. Both stars show clear velocity variations centered on the cluster mean value of approximately $+84 \mathrm{~km} \mathrm{~s}^{-1}$. The two $\gamma$ Doradus stars V20 and V24, for which we have Giraffe spectra, have constant velocities over the 15 epochs, with values near the cluster mean. They are thus cluster members, as are V4, V5 and V9. For V21, long-term RV variations are possibly present, with values between +70 and $+90 \mathrm{~km} \mathrm{~s}^{-1}$, but further analysis is needed before any firm conclusions can be drawn about the membership status and possible dublicity of this object.

\section{Summary and conclusions}

Our two-colour, dual-site photometric campaign from La Palma and La Silla was a success as we have quadrupled the number of known variables in the open cluster NGC 2506. Based on our results we conclude that the cluster contains (at least) 6 oscillating BS stars, $15 \gamma$ Doradus stars, and 6 eclipsing binaries.

Prior to this work, only three short-period variables were known in NGC 2506, and only one of them was classified as a oscillating BS star. We now know 6 oscillating BS stars, of which 5 are multi-periodic. One BS star (V3) shows both shortand long-period variability. To our knowledge, this would be the first BS star for which both p-mode and g-mode oscillations are discovered. Given the fact that we detected 11 oscillations frequencies in this star, makes this blue straggler a very promising target for follow-up research. We therefore reached our goal of data gathering and selection of interesting targets suitable for future investigation. We finally mention that a preliminary mode identification seems to indicate that in four of the BS stars the fundamental radial mode is excited, and for one star (V6) there is evidence of the fundamental and first overtone frequencies being excited.

We report the discovery of $15 \gamma$ Doradus stars of which most are multiperiodic, and at least 14 of them are bona fide $\gamma$ Doradus stars, while one is a prime $\gamma$ Doradus candidate. The $(b-y)$ colours, $M_{V}$ and the $\delta c_{1}, \delta m_{1}$ indices agree well with the corresponding values for the known $\gamma$ Doradus variables.

We have compared frequencies, amplitude ratios and phase differences of the $\gamma$ Doradus stars to state-of-the-art $\gamma$ Doradus models. The results of this comparison are that 1) the lowest, detected frequencies (below $0.25 \mathrm{~d}^{-1}$ ) are predicted to be stable in the models, 2) we observe positive $I-B$ phase differences in disagreement with the models, and 3 ) the majority of the observed frequencies appear not to be dipole modes, contrary with what is expected, as cancellation effects are stronger for modes of higher degree. These results clearly ask for further examination. We plan a forthcoming theoretical study and additional spectroscopic data to investigate the effect of stellar rotation on the observables.

We have for the first time determined periods for the two detached, eclipsing binaries V4 and V5, and preliminary spectroscopic results confirms cluster membership for both.

All these results combined strongly confirm and increase our interest in NGC 2506 as an excellent target cluster for studying $\mathrm{BS}$ and $\gamma$ Doradus stars. 
Acknowledgements. The Danish Natural Science Research Council, the Fund for Scientific Research, Flanders, and the Instrument Center for Danish Astrophysics (IDA) are acknowledged for financial support. J.D.R. and M.R. are postdoctoral fellows of the Fund for Scientific Research, Flanders, and F.G. acknowledges financial support from IDA. This research has made use of the SIMBAD database, operated at CDS, Strasbourg, France.

\section{References}

Alonso, A., Arribas, S., \& Martinez-Roger, C. 1996, A\&A, 313, 873

Arentoft, T., Kjeldsen, H., Nuspl, J., et al. 1998, A\&A, 338, 909

Arentoft, T., Bouzid, M. Y., Sterken, C., et al. 2005, PASP, 117, 601

Breger, M. 2000, Proceedings of Delta Scuti and Related Stars, ed. M. Breger, \& M. H. Montgomery, ASP Conf. Ser., 210, 3

Breger, M., Pamyatnykh, A. A., Pikall, H., \& Garrido, R. 1999, A\&A, 341, 151 Carretta, E., Bragaglia, A., Gratton, R. G., \& Tosi, M. 2004, A\&A, 422, 951

Chen, W. P., Chen, C. W., \& Shu, C. G. 2004, AJ, 128, 2306

Chiu, L.-T. G., \& van Altena, W. F. 1981, ApJ, 243, 827

Claret, A. 2000, A\&A, 363, 1081

Crawford, D. L. 1975, AJ, 80, 955

De Marco, O., Lanz, T., Ouellette, J. A., et al. 2004, ApJ, 606, 151

Dupret, M.-A., Grigahcéne, A., Garrido, R., et al. 2004, A\&A, 414, L17

Dupret, M.-A., Grigahcéne, A., Garrido, R., et al. 2005a, A\&A, 435, 927

Dupret, M.-A., Grigahcéne, A., Garrido, R. et al. 2005b, MNRAS 360, 1143

Ferraro, F. R., Beccari, G., Rood, R. T., et al. 2004, ApJ, 603, 127

Ferraro, F. R., Sabbi, E., Gratton, R., et al. 2006, ApJ, 647, 53

Frandsen, S., \& Kjeldsen, H. 1993, Inside the stars, Proc. 137th IAU Coll., ed. W. W. Weiss, \& A. Baglin, ASP Conf. Ser., 40, 746

Garrido, R. 2000, Proceedings of Delta Scuti and Related Stars, ed. M. Breger, \& M. H. Montgomery, ASP Conf. Ser., 210, 67

Gilliland, R. L., \& Brown, T. M. 1992, AJ, 103, 1945

Gilliland, R. L., Bono, G., Edmonds, P. D. et al. 1998, ApJ, 507, 818

Gratton, R. 2000, in Stellar Clusters and Associations: Convection, Rotation and Dynamos. ed. R. Pallavicini, G. Micela, \& S. Sciortino, Proc. from ASP Conf., 198, 225

Grigahcéne, A., Dupret, M.-A., Gabriel, M., et al. 2005, A\&A, 434, 1055
Grundahl, F., Stetson, P. B., \& Andersen, M. I. 2002, A\&A, 395, 481 Grundahl, F., Arentoft, T., Bruntt, H. et al. 2006, MmSAI, 77, 433

Handler, G. 1999, MNRAS, 309, L19

Handler, G. 2003, Baltic Astron., 12, 253

Handler, G., \& Shobbrook, R. R. 2002, MNRAS, 333, 251

Henry, G. W., \& Fekel, F. C. 2005, AJ, 129, 2026

Henry, G. W., Fekel, F. C., \& Henry, S. M. 2005, AJ, 129, 2815

Hills, J., Day, C. 1976, Astro. Lett., 17, 87

Joshi, S., Mary, D. L., Martinez, P. et al. 2006, A\&A, 455, 303

Kaye, A. B., Handler, G., Krisciunas, K. et al. 1999, PASP, 111, 840

Kim, S.-L., Chun, M.-Y., Park, B.-G., et al. 2001, AcA, 51, 49

Kharchenko, N. V., Piskunov, A. E., Röser, S., et al. 2005, A\&A, 438, 1163

Kjeldsen, H., \& Frandsen, S. 1992, PASP, 104, 413

Kjeldsen, H., Arentoft, T., Bedding, T. R. et al. 1998, in Structure and Dynamics of the Interior of the Sun and Sun-like stars, SOHO 6/GONG 98 Workshop abstract, June 1-4, 1998, Boston, Massachusetts, 385

Kurucz, R. L. 1993, ATLAS9 Stellar Atmosphere programs and $2 \mathrm{~km} \mathrm{~s}^{-1}$ grids, Kurucz CD-ROM No. 13

Lejeune, T., Cuisinier, F., \& Buser, R. 1998, A\&AS, 130, 65

Mapelli, M., Sigurdsson, S., Ferraro, F. R., et al. 2006, MNRAS, 373, 361

McCrea, W. H. 1964, MNRAS, 128, 147

Petersen, J. O., \& Christensen-Dalsgaard, J. 1996, A\&A, 312, 463

Petersen, J. O., \& Christensen-Dalsgaard, J. 1999, A\&A, 352, 547

Poretti, E. 2003, A\&A, 409, 1031

Porter, J. M., \& Townsend, R. H. D. 2005, ApJ, 623, 129

Rowe, J. F., Matthews, J. M., Cameron, C. et al. 2007, Comm. in Asteroseismology University of Vienna

[arXiv: astro-ph/0610640], 148

Salaris, M., Weiss, A., \& Percival, S. M. 2004, A\&A, 414, 163

Sills, A., Adams, T., \& Davies, M. B. 2005, MNRAS, 358, 716

Sills, A., Faber, J. A., Lombardi, J. C. et al. 2001, ApJ, 548, 323

Sperl, M. 1998, Comm. in Asteroseismology, University of Vienna, 111, 1

Tian, B., Deng, L., Han, Z., \& Zhang, X. B. 2006, A\&A, 455, 247

Viskum, M., Kjeldsen, H., Bedding, T. R. et al. 1998, A\&A, 335, 549

Xin, Y., \& Deng, L. 2005, ApJ, 619, 824

Zima, W., Wright, D., Bentley, J., et al. 2006, A\&A, 455, 235 